\title{
Calibration and Data Analysis for the KC1F Fast Magnetics System
}

\author{
R.F. Heeter, A.F. Fasoli, S. Ali-Arshad, J.M. Moret
}

\section{March 1, 2000}

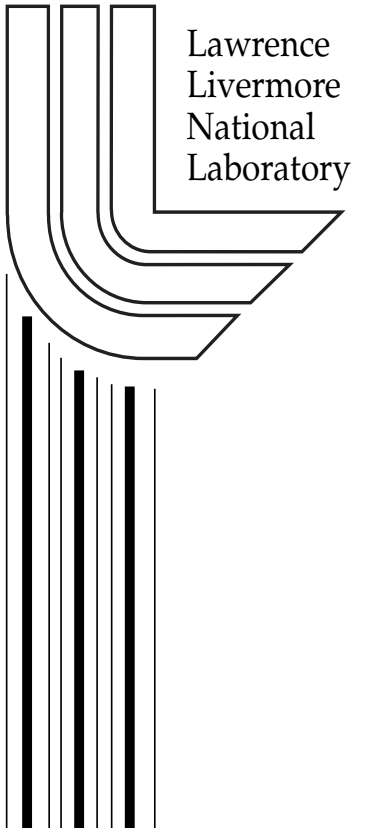




\section{DISCLAIMER}

This document was prepared as an account of work sponsored by an agency of the United States Government. Neither the United States Government nor the University of California nor any of their employees, makes any warranty, express or implied, or assumes any legal liability or responsibility for the accuracy, completeness, or usefulness of any information, apparatus, product, or process disclosed, or represents that its use would not infringe privately owned rights. Reference herein to any specific commercial product, process, or service by trade name, trademark, manufacturer, or otherwise, does not necessarily constitute or imply its endorsement, recommendation, or favoring by the United States Government or the University of California. The views and opinions of authors expressed herein do not necessarily state or reflect those of the United States Government or the University of California, and shall not be used for advertising or product endorsement purposes.

Work performed under the auspices of the U. S. Department of Energy by the University of California Lawrence Livermore National Laboratory under Contract W-7405-Eng-48.

This report has been reproduced directly from the best available copy.

Available to DOE and DOE contractors from the

Office of Scientific and Technical Information

P.O. Box 62, Oak Ridge, TN 37831

Prices available from (423) 576-8401

http://apollo.osti.gov/bridge/

Available to the public from the National Technical Information Service

U.S. Department of Commerce 5285 Port Royal Rd., Springfield, VA 22161 http://www.ntis.gov/

OR Lawrence Livermore National Laboratory Technical Information Department's Digital Library http://www.llnl.gov/tid/Library.html 


\title{
Calibration and Data Analysis for the KC1F Fast Magnetics System
}

\author{
R. F. Heeter* \\ Princeton Plasma Physics Laboratory \\ P.O. Box 451; Princeton, NJ 08543 \\ A. F. Fasoli \\ Plasma Science and Fusion Center, NW17-254; Massachusetts Institute of Technology \\ 175 Albany St.; Cambridge, MA 02139 \\ S. Ali-Arshad ${ }^{\dagger}$ \\ JET Joint Undertaking; Abingdon, Oxfordshire, OX14 3EA; UK \\ J. M. Moret \\ CRPP-EPFL, CH-1015 Lausanne, Switzerland
}

(March 1, 2000)

\begin{abstract}
Alfvén Eigenmodes (AEs) and other magnetohydrodynamic (MHD) phenomena have been studied at the Joint European Torus (JET) using a new 8-channel, 4 s, 1 MHz, 12-bit data acquisition system "KC1F" in conjunction with the JET fast Mirnov magnetic fluctuation pickup coils. The JET magnetic pickup coils were calibrated for the first time in the range $30-460 \mathrm{kHz}$ using a new remote calibration technique which accounts for the presence of the first few LRC circuit resonances. A data-processing system has been developed within the MATLAB software environment to produce spectrograms of fluctuation amplitude and toroidal mode number versus frequency and time. The analysis software has been automated to allow routine overnight production of spectrogram web pages. Modes with amplitudes $\delta B / B \geq 10^{-8}$ and toroidal mode numbers $|n|<32$ are now routinely detected. A pulse-characterization database has also been developed to select for the analysis of various useful subsets of the 4000+ JET discharges for which KC1F data is now available. Based on the work presented here and recent advances in data-acquisition technology, it should now be possible to obtain complete diagnostic data on the AEs.
\end{abstract}

\section{INTRODUCTION}

This paper provides a comprehensive discussion regarding a set of diagnostic and data analysis tools developed to study the Alfvén Eigenmodes (AEs) and other high-frequency (up to $500 \mathrm{kHz}$ ) magnetohydrodynamic (MHD) instabilities at the Joint European Torus (JET). The AEs and related modes are of interest for fusion research because they may be resonant with the energetic ions produced by fusion reactions or auxiliary heating, and sufficiently strong pressure gradients in the resonant ions may drive the modes unstable to the point where the fast ions are ejected from the plasma. AE studies at JET are of particular interest due to the substantial alpha particle populations which can be produced in JET D-T plasmas with up to $16 \mathrm{MW}$ of fusion power and near-breakeven conditions $Q \equiv P_{\text {fus }} / P_{\text {heat }} \approx 2 / 3 .^{1,2}$ Readers interested in AE physics and relevant fluctuation diagnostic techniques are referred to a recent review article ${ }^{3}$ and references therein. A physics-focused version of this paper, which leaves out many of the technical details, is also being published in the refereed literature ${ }^{4}$ A more comprehensive discussion of the $\mathrm{KC1F}$ system in the context of Alfvén Eigenmode research at JET, including the KC1F software manuals and operational procedures, is included in the author's doctoral thesis. ${ }^{5}$ Several results obtained with the KC1F diagnostic at JET have appeared in the literature. ${ }^{1,2,5-17}$

In this paper, Section II begins with theoretical considerations which set the context for the system design, and then presents the system specifications in the context of other JET diagnostic and data acquisition systems. Section III details the calibration of the $\mathrm{KC1F}$ system, which is subdivided into an amplifier/digitizer section and a probeand-cable section. The calibration of the probe-and-cable section required the development of a new procedure and was the first remote, absolute calibration of the JET fast magnetic probes in the frequency range of interest for AE studies $(30-460 \mathrm{kHz})$. Both the theory and application of the procedure are presented. Supplementary technical

*Present Address: Lawrence Livermore National Lab; P.O. Box 808; Livermore, CA 94551.

${ }^{\dagger}$ Present Address: CERN; CH-1211, Geneva 23; Switzerland. 
details of the calibration are presented in Appendix A, including results from benchmarking the procedure using a test circuit. With the calibration in hand, Section IV details the data processing system, including the hardware pipeline, the software developed to apply the calibration to the data via digital filtering, and the software developed to analyze the calibrated data. Selected experimental results ${ }^{5}$ obtained using these tools are included as examples. Section $\mathrm{V}$ describes the pulse-characterization database developed to assist in mining the large KC1F database and allow systematic analyses of AE phenomena in various JET plasma configurations.

\section{SYSTEM DESIGN AND SPECIFICATIONS}

\section{A. Theoretical Considerations}

The primary use of the KC1F system is to study the magnetic fluctuations induced by Alfvén Eigenmodes and other MHD activity in the same frequency range. Since a review article on AE physics has recently been published, ${ }^{3}$ here we simply highlight selected aspects of AE physics relevant to AE measurement and analysis, using the JET parameters to set the context for the $\mathrm{KC} 1 \mathrm{~F}$ system.

The standard dispersion relation for the Alfvén wave spectrum for infinite homogeneous plasmas is obtained from the ideal MHD equations ${ }^{18,19}$ or from the general cold-plasma dispersion relation: ${ }^{20}$

$$
\begin{aligned}
\omega^{2} & =k_{\|}^{2} v_{A}^{2}, \\
v_{A} & =B / \sqrt{4 \pi \sum_{\text {ions }} m_{i} n_{i}},
\end{aligned}
$$

for $\omega \ll \Omega_{i}$, in CGS units, with $B$ as the magnetic field, $m_{i}$ as the mass of each type of ion, and $n_{i}$ for the density of each ion species. In this geometry the Alfvén spectrum is a simple continuum, but as one moves to more tokamak-like parameters the spectrum becomes more complex.

For an inhomogeneous periodic cylinder with an axial current, the axial and poloidal periodicity can be Fourieranalyzed, and the spectrum for each flux surface breaks into a set of discrete modes. Because of the radial profiles of density and field, for each mode $k_{\|} \rightarrow k_{\|}(r)$ and $v_{A} \rightarrow v_{A}(r)$. The variation of the plasma parameters allows a smooth and continuous variation of the shear Alfvén frequency $\omega$, and for any given frequency one can typically find at least one shear Alfvén wave somewhere in the plasma with that frequency. Because of this property the shear Alfvén spectrum is still known as the Alfvén continuum (in frequency).

However, if the functions $k_{\|}(r)$ and $v_{A}(r)$ satisfy

$$
k^{\prime} / k=-v_{A}^{\prime} / v_{A}
$$

(with $k^{\prime}$ denoting the radial derivative of $k$, and likewise for $v_{A}$ ) then a plot of the function $\omega\left(k_{\|}\right)$vs. $r$ will have a minimum, and below this minimum frequency there is a "gap", a frequency range where no shear Alfvén waves exist. But within the gap the MHD equations admit new eigenmode solutions, the Global Alfvén Eigenmodes (GAEs). ${ }^{21,22}$ Depending on the plasma profiles the Alfvén gap may extend across the entire plasma, and aside from the GAEs there may be no Alfvén waves in that frequency range.

In tokamak plasmas, additional geometric effects such as toroidal field curvature and elliptical and triangular poloidal cross-sections also alter the mode spectrum, coupling nearby poloidal harmonics together and introducing additional Alfvén gaps in the continuous Alfvén frequency spectrum. Within these gaps, new classes of Alfvén Eigenmodes (AEs) can be found. All the Alfvén Eigenmodes differ from the shear Alfvén waves in having a global rather than local structure.

\section{TAEs}

In present-day tokamak experiments the TAE is the canonical, most commonly studied AE. The principal Alfvén gap in a tokamak arises because of the finite radius of the torus. Inside this gap the TAE, or toroidicity-induced Alfvén Eigenmode, ${ }^{23,24}$ exists as a solution to the wave equation. The central frequency of the TAE gap (in cgs units) sits at

$$
f_{T A E}=v_{A} / 2 q R \sim B_{0} / q \sqrt{\sum_{i o n s} m_{i} n_{i}} .
$$


Experimentally the TAE frequency is measured from outside the plasma and therefore includes a Doppler shift induced by the rotation of the plasma:

$$
f_{\text {meas }}=f_{T A E}+n f_{\text {rot }}
$$

where $\mathrm{n}=$ toroidal mode number and $f_{\text {rot }}$ is the toroidal rotation frequency. In principle there can also be a Doppler shift from poloidal rotation, but empirically (in JET at least) this appears so far to be negligible.

The actual frequency of a TAE will be somewhere within the gap, whose width is on the order of the inverse aspect ratio at the radial location of the gap, $\Delta f / f \approx \epsilon \ll 1$. Thus the gap frequency is usually a reasonable estimate of the correct TAE frequency, and both the TAE frequency and the gap frequency scale similarly with magnetic field and ion density. In calculating $f_{T A E}$, the parameters $n_{i}$, q, and $f_{r o t}$ should be evaluated at the location of the mode, typically at $r / a \approx 0.3$ where the fast ion pressure gradient is normally steepest. We have found that for standard JET plasmas with conventional current profiles, a good quick estimate for TAEs with $0<n<12$ can be obtained using $q=1.3$ to 1.5 and the line-averaged electron density $\bar{n}_{e} .5,8$

2. Other types of AEs: EAEs, BAEs, kTAEs, etc.

Other plasma shaping effects also cause the Alfvén spectrum to develop gaps containing eigenmodes. In particular, tokamaks such as JET with poloidal divertors have both ellipticity and triangularity in the poloidal cross-section. There are Ellipticity-induced Alfvén Eigenmodes (EAEs) in a gap at

$$
f_{E A E} \approx 2 f_{T A E},
$$

and Triangularity-induced (non-circular) modes (NAEs) at

$$
f_{N A E} \approx 3 f_{T A E} .
$$

Additional modes have also been predicted to exist below the TAE frequency, resulting from coupling between shear Alfvén waves and lower-frequency modes such as acoustic waves.

\section{AE Mode Structure}

The precise theoretical determination of the frequency and structure of particular modes requires sophisticated computer codes such as NOVA-K, CASTOR, MISHKA, or PENN. ${ }^{25,11,26}$ These take as inputs the magnetic field equilibrium $(B, q)$ and density profiles, and then use either MHD, hybrid MHD-kinetic or gyrokinetic models to determine the mode frequency and structure. The radial, toroidal and poloidal structure are all important. Typically a given $\mathrm{AE}$ has a single toroidal mode number, $\mathrm{n}$, but consists of multiple poloidal harmonics. Multiple AEs can exist at the same time, with the same $\mathrm{n}$ but different structure and slightly different frequency. The radial structure can be localized or extended. In the poloidal plane the structure of different AE modes can have a ballooning (larger on the outboard side) or anti-ballooning (larger on the inboard side) character. The modeling results are sensitive to both the model equations and the plasma equilibrium information imported into the model. The models allow a determination of the mode frequency at the onset of linear instability, but once the modes grow, nonlinear saturation effects can cause the mode frequency to drift. ${ }^{27}$

\section{AE Drive}

As with other plasma waves, the stability of AEs depends on the balance between driving and damping terms. The AEs are of particular interest because often they are not as heavily damped as the waves in the shear Alfvén continuum. It has been realized for some time that Alfvén waves might be destabilized by resonant ions in magnetically confined plasmas. ${ }^{28,29}$ This is true of the AEs as well, and the possibility of AE excitation by alpha particles ${ }^{30}$ raises $^{2}$ the worry that fast-ion driven AEs might cause transport and loss of the alphas, possibly impeding reactor ignition ${ }^{31}$ or inducing wall damage if losses exceed $5 \%$ overall. ${ }^{32}$ This has made AE physics an important topic in fusion research.

AEs can resonate with the characteristic frequencies of both passing and trapped particles. In JET plasmas the resonance conditions ${ }^{3}$ can be satisfied by injected neutral beam ions (at relatively low toroidal fields), ICRF-heated ion tails, and the alpha particles produced by fusion reactions. Provided the mode drive (arising from the radial pressure gradient of the resonant fast particle population) exceeds the sum of various damping terms, ${ }^{3}$ the modes may be driven unstable, and it is these destabilized modes which the KC1F system seeks to diagnose. 


\section{Nonlinear AE Saturation and Particle Transport}

Since AEs are resonant with energetic ions, sufficiently large AEs can cause transport and possibly losses of the ions. Loss fractions greater than a few percent become troublesome for ignition and near-ignition reactor designs such as ITER. ${ }^{33}$ The importance of the fast-ion loss issue was highlighted at TFTR when localized losses attributed to TAE-induced ripple trapping of ICRH tail ions caused melting of debris shields on the bottom of the vacuum vessel. ${ }^{3,32}$ In order to assess fast ion losses arising from the AEs one must understand both the linear (existence and stability) and nonlinear (saturation and particle transport) physics of AEs. The same knowledge is also a prerequisite for using the AEs for alpha particle control. ${ }^{34,35}$ In order to understand their nonlinear dynamics it is therefore desirable to be able to measure the amplitudes of individual AEs as a function of time, with temporal resolution comparable to the linear growth time $(1 / \gamma)$ of the modes (with $10^{-3}<\gamma / \omega<10^{-1}$ being typical).

It is expected on theoretical and experimental grounds that mode amplitudes above a stochastic threshold

$$
(\delta B / B)_{\text {thresh }} \approx 5-50 \times 10^{-4}
$$

will enter strongly nonlinear regimes with substantial fast ion transport. ${ }^{31,27,11}$ Thus one also needs AE diagnostics to be sensitive to mode amplitudes which are less than or comparable to this level. It should be noted that mode amplitudes measured at the edge may be up to 3 orders of magnitude weaker than the peak amplitude in the core, as will be discussed in Section II D.

\section{B. Measurement Objectives and Design Compromises}

JET has a number of diagnostic systems which can in principle detect Alfvén Eigenmodes. The modes may be detected by their perturbations to the plasma magnetic field, density, and electron temperature. Many methods for measuring these parameters exist, ${ }^{36,37}$ and several are implemented at JET. However, there are always practical limitations on what one can measure, and it is worth characterizing the AE data acquisition problem in order to understand the diagnostic methods used at JET.

To fully characterize the AEs, one would ideally like to determine routinely their time-dependent toroidal, poloidal and radial structure and amplitude. At JET this is only just now becoming feasible from the data acquisition perspective. The amount of data, D that is needed can be expressed as

$$
D=t_{s} f_{s} N_{c} N_{b} N_{p}
$$

where $t_{s}$ is the duration of the sampling window per pulse, $f_{s}$ is the sampling frequency, $N_{c}$ is the number of channels needed, $N_{b}$ is the number of bytes recorded per sample, and $N_{p}$ is the number of pulses to be recorded.

The main heating phase of JET discharges lasts typically 10 seconds, so we take $t_{s}=10$ seconds. The AE frequency range in JET (from Equation 4) extends from $50 \mathrm{kHz}$ for TAEs at $B_{T}=0.8$ Tesla to over $1 \mathrm{MHz}$ for NAEs at $B_{T}=3.8$ Tesla, so one would like to have $f_{s} \geq 2 \mathrm{MHz}$ to resolve the AEs. To obtain a comprehensive picture of AE behavior in a tokamak of 3 meter major radius and 1 meter minor radius would require at a bare minimum 10 radial channels, 5 poloidal channels and 5 toroidal channels, for a total of $N_{c}=20$ channels. As only the toroidal structure of the AEs is reasonably simple, greater numbers of radial and poloidal channels would be very useful. One would also like good signal-to-noise ratios as well, so $N_{b}=2$ bytes per sample (12 or 16 bits) are necessary. JET produced roughly $N_{p}=4000$ pulses in 1997 . The result of all this is a minimum of $D=3.2 \times 10^{12}$ bytes of information. Even this minimum exceeds the total JET data acquisition from the first plasma in 1983 through the end of $1997 .{ }^{38}$ On a per-pulse basis one would need upwards of $800 \mathrm{MB}$ of data, which is more than double the typical 1997 per-pulse JET data acquisition. ${ }^{38}$ This will be possible in timescales on the order of a few years given projected advances in data storage technology, but for the work described here it was necessary to make compromises. In fact, each of the relevant diagnostic data acquisition systems at JET can be seen as representing the results of different compromises.

\section{AE Synchronous Detection}

The synchronous detection system is used in conjunction with the saddle coils, allowing measurements of the amplitude and phase response of plasma parameters to saddle coil-driven fluctuations. The fundamental compromise which this system makes is to minimize the bandwidth that is sampled. This system has 32 channels of data acquisition, synchronized to the frequency of the function generator used in the saddle coil system. The system allows a comprehensive picture of fluctuations occurring at the single, swept frequency to which it is tuned. The data output is 
sampled at relatively slow rates $(1 \mathrm{kHz})$ for 12 seconds. This allows real-time detection of resonant plasma responses, including damping measurements for the low-n AEs which the saddle coils can excite. This has had a considerable impact for $\mathrm{AE}$ experimental work and benchmarking of $\mathrm{AE}$ theory. ${ }^{39-41,3}$

\section{2. $C A T S$}

A second major data acquisition system relevant to AE studies at JET is the Central Acquisition and Trigger System, CATS. One of the purposes of CATS is to study fluctuations leading up to disruptions. The main compromise made by CATS is to reduce the duration of the sampling period. It has a large number of channels with data provided at moderate sample rates $(250$ to $500 \mathrm{kHz}$ ), but typically for only short intervals totalling 0.2 to 0.5 seconds per pulse. $^{42}$ This is partially compensated by partitioning the sample windows to allow short "peeks" at various times in the discharge. Because of the large number of channels, CATS is able to take detailed snapshots of the plasma at particular times. It is worth noting that the sampling rates and sample windows have been progressively increasing with time as the price/performance ratio of the relevant hardware improves, and this system will be of great use to future AE studies.

\section{3. $K C 1 F$}

The work reported here focuses on a third major data acquisition system, known as "KC1F" in the JET nomenclature. This system represents a balanced compromise, having a moderately limited number of channels (8), a moderately limited sample window (4 seconds), and a moderate reduction in sample rate $(1 \mathrm{MHz})$, all of which still allow routine measurements of TAEs and often EAEs and NAEs. 12 bits of data are taken per sample point, but 2 bytes of storage is used. This results in the collection of $64 \mathrm{MB}$ of data per pulse, which was just barely practical at JET in the 1997-8 timeframe, and apparently represented over $10 \%$ of the data collected in $1997 .{ }^{38}$

\section{Diagnostic Signal Selection for KC1F}

Although most of the JET diagnostics have bandwidth limitations that prevent useful information from being obtained in the $100 \mathrm{kHz}-1 \mathrm{MHz}$ frequency range, several diagnostics used in the 1997-1998 campaigns produced signals that could conceivably be used to detect the AEs and other interesting phenomena. These included the fast magnetic Mirnov probes, a small subset of the soft x-ray diode arrays, $D_{\alpha}$ emission, and the x-mode correlation reflectometer. Although not sufficient for fully characterizing AEs, these were able to yield several novel and interesting results. ${ }^{5}$ Although one would ideally like radially-resolved core measurements of the AEs, thus making soft x-rays and reflectometry preferable, in practice edge measurements with magnetic probes proved to be the simplest and most sensitive method for detecting the AEs using the KC1F digitizers. More recently the $\mathrm{x}$-mode reflectometer signals have also shown evidence of AEs, albeit with lower signal-to-noise ratios. ${ }^{5}$

\section{KC1F Fast Magnetics System Specifications}

The hardware for the JET fast magnetics system "KC1F" consists of a set of magnetic pickup coils (Mirnov probes) hooked up to a fast-sampling analog-to-digital converter (ADC) connected to a PC which is linked into the JET COntrol and Data Acquisition System (CODAS). (Several of the ADC channels are now digitizing X-mode reflectometer signals, but that is beyond the scope of this paper.) A graphical overview of the entire system is provided by Fig. 1; here we focus on the first two stages, the probes and data acquisition system. 


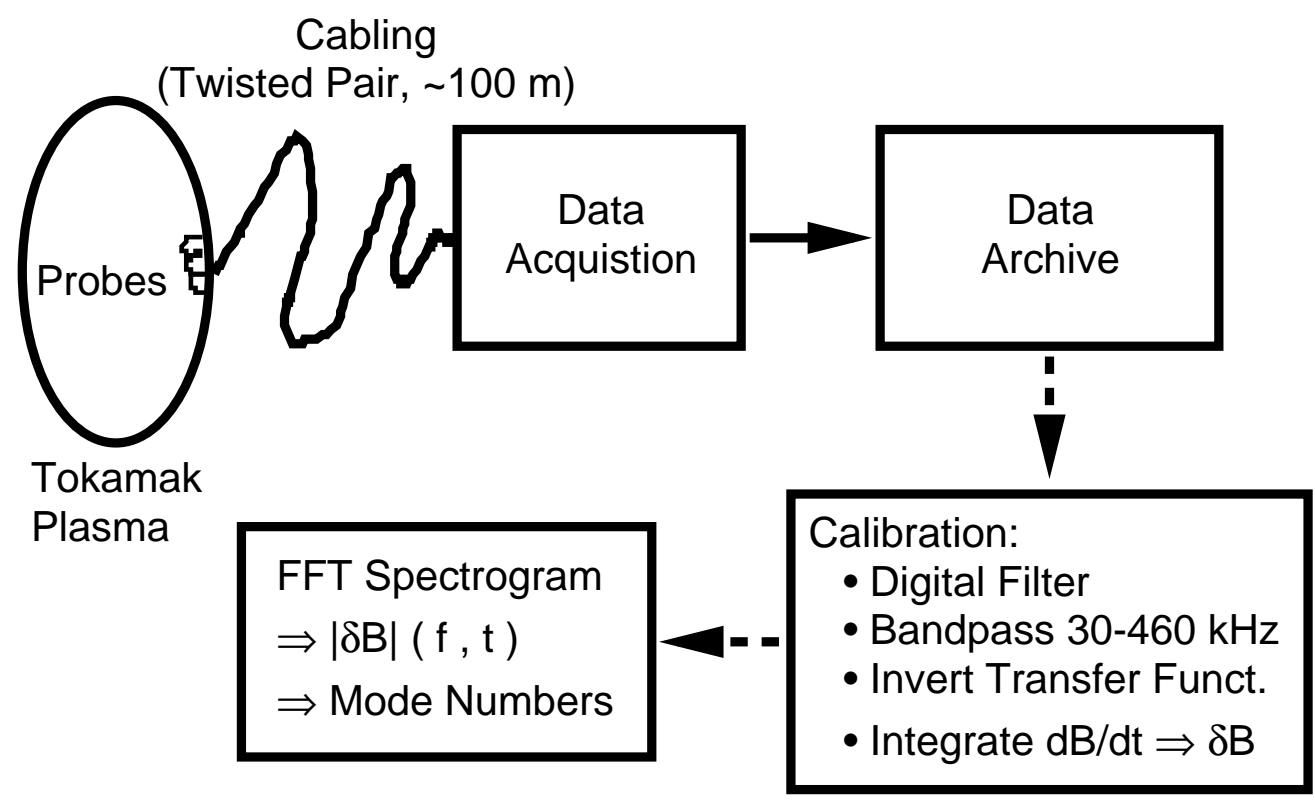

FIG. 1. Overview of $\mathrm{KC} 1 \mathrm{~F}$ data acquisition and processing.

JET has both toroidal and poloidal coil arrays designed to measure fluctuations in the poloidal magnetic field with good response up to at least $500 \mathrm{kHz}$. Both arrays may be divided into sets with low and high resolution in position space. Thus far KC1F has only been connected to probes from the toroidal sets, which sit somewhat above the midplane on the outboard side of the torus.

The 10-probe low-resolution toroidal array spans the full circumference of the torus with 10 probes, allowing determination of low-n mode numbers. The high-resolution array is a set of 3 closely-spaced probes, which allows one to determine the toroidal structure of high-n modes $(|n|<32)$ as discussed in Section IV C. Poloidal magnetic fluctuation amplitudes (at the probe) as low as $\left|\delta B_{\theta}\right|>3 \times 10^{-8}$ Tesla can be measured from the calibrated probe signals, corresponding to normalized amplitudes $|\delta B / B|>1 \times 10^{-8}$.

Each of the JET fast magnetics Mirnov probes is essentially a solenoid coil with length $10 \mathrm{~cm}$, diameter $4 \mathrm{~cm}$, and about 60 turns. For an ideal coil exposed to a time-varying magnetic field $d B / d t$, the output voltage can be readily derived from Faraday's Law, and follows

$$
V=N A d B / d t
$$

where $N$ is the number of turns, $A$ is the area enclosed by a single turn, and therefore $N A$ is the effective probe area. $^{36}$ The JET probes have an effective area $N A=0.064 \mathrm{~m}^{2}$. The probes are measured to have a DC resistance of $50 \Omega$ at the typical JET vacuum vessel operating temperature of $320^{\circ} \mathrm{C}$. Basic information about each of the toroidal coils is given in Table I. ${ }^{43}$

\begin{tabular}{ccccc}
\hline \hline Name & Set & Tor. Angle. $\Phi$ & Eff. Area & Status $1997-1998$ \\
\hline T1 & Low-Res & $48.0^{\circ}$ & $0.064 \mathrm{~m}^{2}$ & 0.064 \\
T2 & Low-Res & 87.15 & 0.064 & Okay \\
T3 & Both & 122.0 & 0.064 & Okay \\
H302 & High-Res & 137.94 & 0.064 & Okay \\
H303 & 148.11 & 0.064 & Okay \\
H304 & High-Res & 153.74 & 0.064 & Okay \\
T4 & Both & 155.38 & 0.064 & Okay \\
T5 & Low-Res & 192.83 & 0.064 & Dead \\
T6 & Low-Res & 227.9 & 0.064 & Unreliable \\
T7 & Low-Res & 267.2 & 0.064 & Okay \\
T8 & Low-Res & 302.1 & 0.064 & Okay \\
T9 & Low-Res & 335.4 & 0.064 & Okay \\
T10 & Low-Res & 12.87 & Dead \\
\hline \hline
\end{tabular}

TABLE I. Locations of the JET fast magnetics Toroidal Mirnov coils. The zero reference for the toroidal angles is arbitrary for our purposes. 
The coil signals are cabled out of the torus using individually screened twisted-pair cable which runs into the radiation-shielded diagnostic hall.

The KC1F fast acquisition system consists of 8 Waugh differential buffer amplifiers whose outputs go into a "Falcon" model transient recorder system manufactured by the Sunnyside Corp., which is controlled by a PC system connected to the JET DATANET diagnostic data network. The Waugh buffer amplifiers shield the transient recorder ADCs against transient high voltages from the tokamak plasma and other systems. The Falcon transient recorder system is an 8-channel, 12-bit, transputer-based ADC with $64 \mathrm{MB}$ of onboard RAM. This Sunnyside system may acquire data at either $1 \mathrm{MHz}$ for 4 seconds, or $2 \mathrm{MHz}$ for 2 seconds, although the latter mode of operation is not robust (roughly 1 failure per 7 pulses), apparently because of problems with the trigger signal sent from the central JET control systems. There is nominally only \pm 1 count of noise in the ADC system proper (i.e. 1 part in 2048), which is borne out in practice.

It is important to note that because this system relies on magnetic probe measurements at the outboard edge above the midplane, it may not always be possible to observe strongly core-localized AEs, AEs with a highly anti-ballooning mode structure, or modes with a strong up/down asymmetry favoring the lower part of the plasma. Furthermore, the magnetic fluctuation amplitudes measured at the edge may be up to 3 orders of magnitude smaller than the core amplitudes, according to both theoretical models of the AE mode structure and reports of simultaneous internal and external measurements in other tokamaks. ${ }^{44,3}$

\section{FAST MAGNETICS CALIBRATION AT JET}

\section{A. Statement of the Problem}

In order to determine the amplitudes of modes (such as AEs) detected by the magnetic probes, one must absolutely calibrate the amplitude response vs. frequency of the entire data collection system. To determine toroidal (or poloidal) mode numbers it is also necessary to cross-calibrate the phase response vs. frequency of a set of probes at different toroidal (or poloidal) locations. In the JET case the response curves are non-trivial because the probe circuits are non-ideal: the simple response function in Equation 10 above describes the voltage induced across the probe by a time-varying magnetic field, but the probe has a finite self-inductance and resistance, and it is connected to long cables with their own inductance and capacitance. Circuit resonances therefore alter the voltage that reaches the digitizers compared to that at the probe itself. Additionally, although all of the JET probes are of identical design and virtually identical construction, there are small but unavoidable differences in their cabling and connections from the inside of the torus to the diagnostic digitizers in the diagnostic hall. Consequently, the high-frequency response of these nominally identical probes is not sufficiently identical for our purposes. Furthermore, the amplitude and phase response characteristics of the different digitizer channels need not be identical, so an end-to-end calibration is necessary.

The basic problem in the calibration is identifying the frequency-space complex transfer function which converts the digitized fluctuation signal (in counts) back into the amplitude and phase of a given magnetic field fluctuation at the probe head (in Tesla per second). Because neither the probes nor the KC1F channels are identical, each probe-channel combination must be calibrated independently, and recalibrated whenever the signal connections are changed. Moreover, because the characteristics of the circuit may change over time, the calibration needs to be repeated periodically, and consequently a standardized procedure is desirable.

In principle the "obvious" way to do the calibration is to place a controllable, oscillating magnetic field source near the probes, and directly compare the digitized signals with the known input. However, at JET a direct in situ calibration cannot be performed easily (and certainly not routinely) due to torus access restrictions. This is a problem which is difficult in most tokamaks and is made worse in JET by the radiological safety requirements arising from JET's D-T capability. However, the transfer function for the "Sunnyside" section of the circuit, with the amplifiers and digitizers, can be measured directly by injecting a known signal. Meanwhile, we have developed another method for the probe-and-cable section of the circuit that leads into the torus. The calibration for a given probe is then combined with that for a given Sunnyside channel to yield an overall calibration.

For the probe-and-cable section, instead of a direct calibration, a remote calibration technique has been developed. This technique builds upon the work of one of the authors (Moret) on the TCV tokamak at CRPP-Lausanne, ${ }^{45}$ and makes use of our knowledge of the magnetic probe circuit structure. Basically we measured the circuit impedance vs. frequency and then used the known circuit structure to determine the transfer function, as explained in Section III C.

For $\mathrm{AC}$ circuits it is convenient to work in the Laplace $(s=i \omega)$ frequency-domain space. Here the output signal $\mathrm{S}$ (in digitizer counts) of the fast digitizers is related to the rate of change of the magnetic field $d B / d t$ along the probe 
axis via

$$
S=H_{a}(s) H_{p}(s) \delta B
$$

where $H_{a}$ is the transfer function of the amplifier-digitizer (Sunnyside) section, $H_{p}$ is the transfer function of the probe-cable section (including $N A s$ as the Laplace-space form of Eq. 10), and $\delta B$ is the magnetic fluctuation being measured at the probe. The two transfer functions can individually be represented as $H(s)=B(s) / A(s)$ in the frequency domain, where $\mathrm{B}$ is the output and $\mathrm{A}$ is the input.

The calibration problem reduces to the problem of determining $H_{a}(s)$ and $H_{p}(s)$, but once the calibration transfer function is determined, there remain the problems of applying the calibration to the data and then carrying out systematic studies with data from large numbers of pulses. These challenges are addressed in Sections IV and V, respectively.

\section{B. Calibration of Amplifier-Digitizer Section}

The calibration signal used to directly measure the amplifier-digitizer transfer function $H_{a}$ was injected from a Rohde \& Schwartz function generator set to output sine oscillations. To maximize the signal to noise ratio the amplitude was set as large as possible without saturating the ADCs. For the buffer-amplifier gain settings used, the amplitudes ranged from $500 \mathrm{~mW}$ to $4 \mathrm{~V}$ peak-to-peak for different calibration attempts. The frequency was swept up-down-up from $20 \mathrm{kHz}$ to $500 \mathrm{kHz}$ in 0.5 seconds (1.0 seconds for the full cycle back to $20 \mathrm{kHz}$ ), with the sweep rate chosen to fit one complete unbroken sweep within the digitization period.

The signal was sent through a 1:1 measurement isolation transformer to prevent the formation of ground loops, since the signal generator is grounded whereas the amplifier and digitizer are normally isolated. It was then split 8 ways to arrive in each of the buffer amplifier inputs. The resulting digitized signal for one channel is shown in Fig. 2, from which it is clear that the amplitude response is certainly not constant in frequency. For reference, the amplitude of the injected signal was measured with an oscilloscope and found to be constant to within $2 \%$ over the whole sweep range. (We also tested the response to a $1 \mathrm{kHz}$ triangle waveform and determined that the system response vs. injected voltage was linear at fixed frequency.)

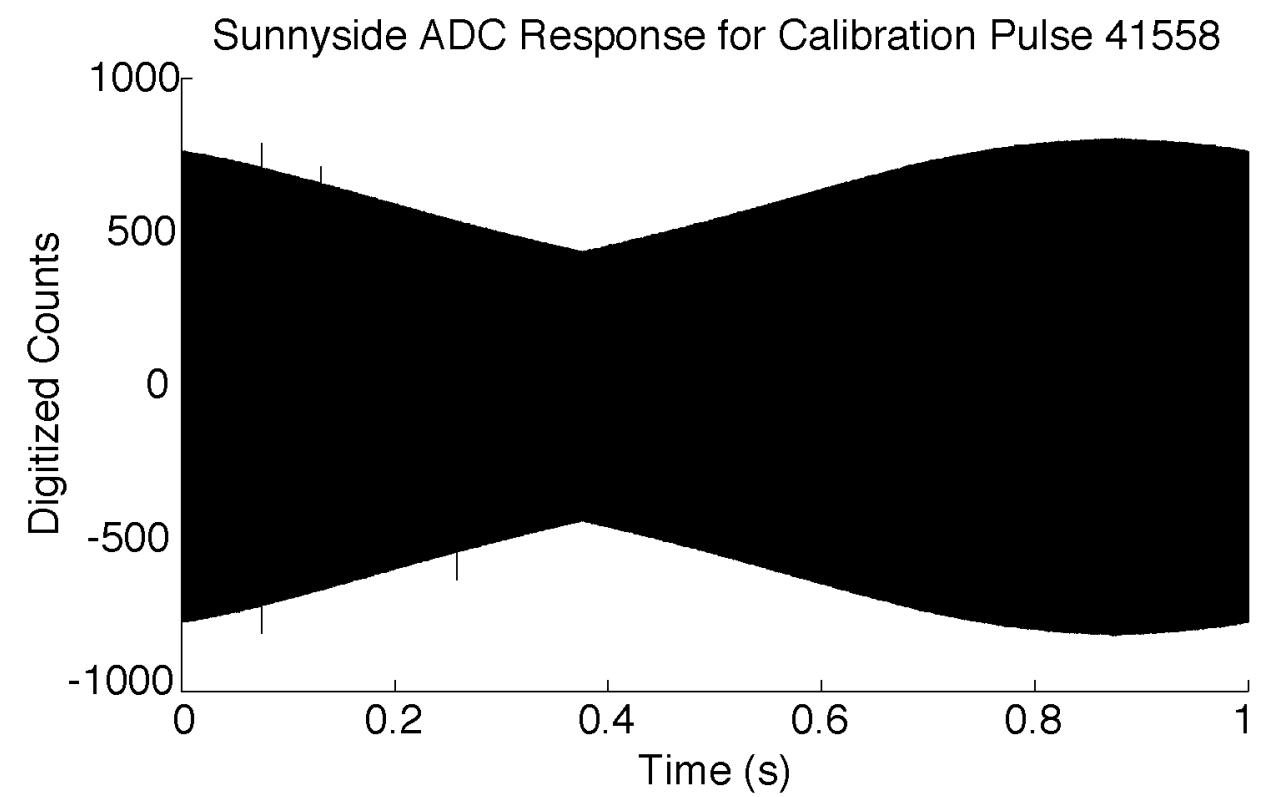

FIG. 2. Typical amplifier-digitizer response to constant-amplitude frequency-swept input. The amplitude varies depending upon the frequency injected at a given time; individual oscillations are not resolved.

The digitized signals were analyzed using software routines written using the technical-computing package MATLAB ${ }^{46}$ including the Signal Processing Toolbox. ${ }^{47}$ The routines determine the absolute amplitude (counts/volt) and relative phase (between channels) of the amplifier-digitizer response to the input signal.

First, the data is divided into a series of short (typically $1.024 \mathrm{~ms}$ ) timeslices, each of which is Fast Fourier Transformed (FFT'd). The frequency of the injected signal in each timeslice is determined from the maximum power 
in the FFT. The FFT phase at the maximum frequency is recorded. The peak-to-peak signal amplitude in the timestep window is converted into counts/volt and also recorded.

For each timestep the mean phase response of each of the ADC channels at the frequency injected at that time is determined, and then for each channel the deviation in phase response of each channel from the mean at that frequency is calculated. The RMS phase deviation of all channels is $<0.1 \mathrm{rad}\left(0.5^{\circ}\right)$ at $50 \mathrm{kHz}$, but increases to about $0.5 \mathrm{rad}\left(2.5^{\circ}\right)$ at $500 \mathrm{kHz}$. This would become a significant source of error for determining mode numbers, so for each channel the phase deviation from the mean is fitted, and this information is later included in the transfer function. The amplitude $(A)$ response is fairly uniform for all channels, as shown in Fig. 3. The fitted phase-deviation $(\delta \phi)$ curves vary, however. This is expected from the RMS phase deviation. The curves are shown in Fig. 4.

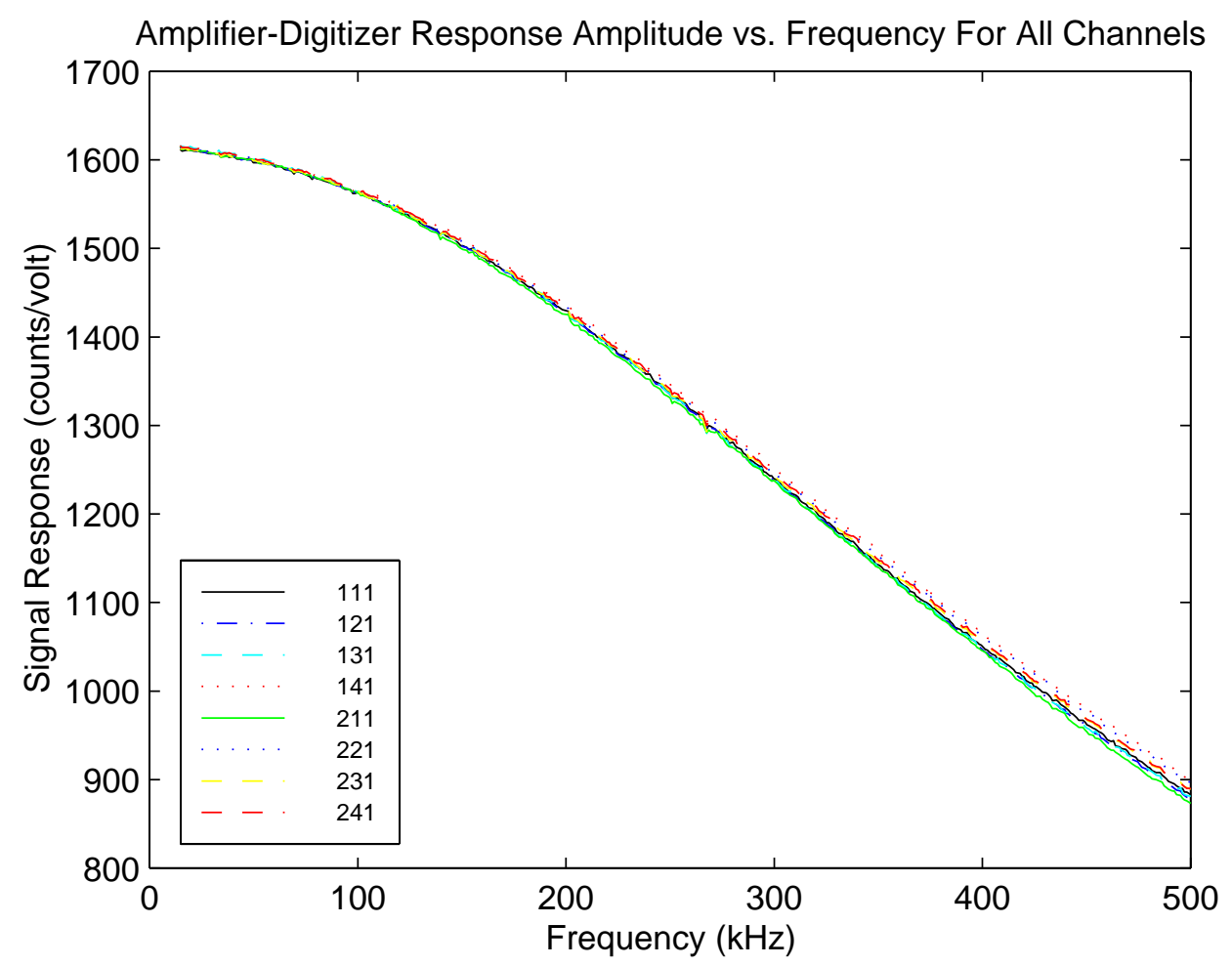

FIG. 3. Amplitude Response of Amplifier-Digitizer Section vs. Frequency

These response curves are converted to a complex response $A(\omega) e^{i \delta \phi(\omega)}$. This is then automatically fit to a Laplacespace $(s=i \omega)$ polynomial of the form $H_{a}(s)=\frac{B(s)}{A(s)}$, with $B(s)$ consisting of a constant gain and a linear phase-drift term, and a quadratic denominator $A(s)$ whose role is mainly to capture the amplitude response vs. frequency. The fit routine used is the sti.m MATLAB function written by one of the authors (Moret). ${ }^{45}$ The polynomial is then compared with the original data and assessed for overall fit quality (error in both amplitude and phase). Typically the maximum error in the amplitude is about $0.5 \%$, with the worst channel being $1.0 \%$. Meanwhile the mean absolute phase noise for each channel is between 0.004 and $0.009 \mathrm{rad}$, i.e. $0.2^{\circ}$ to $0.5^{\circ}$. The coefficients of the polynomials for $H_{a}$ are recorded along with the fit error information.

The calibration process is repeated whenever a gain setting change or other modification is made to the ADCs connected to the magnetic probes. This proved very useful when we upgraded from 1-second to 4-second data acquisition: the calibration results gave bizarre differences in the phase response of the various channels, and by comparing the digitized signals we discovered small, random, discrete shot-to-shot shifts of $1-4 \mu s$ in the relative timing between channels. It turned out that the hardware change to upgrade the system memory had entailed some software changes which accidentally de-synchronized the various channels. ${ }^{48}$ Had this problem not been identified and the channels not been re-synchronized, the information obtained on the relative phase of signals from different channels would have led to severe errors in the toroidal mode number determinations over most of the system's frequency range! 


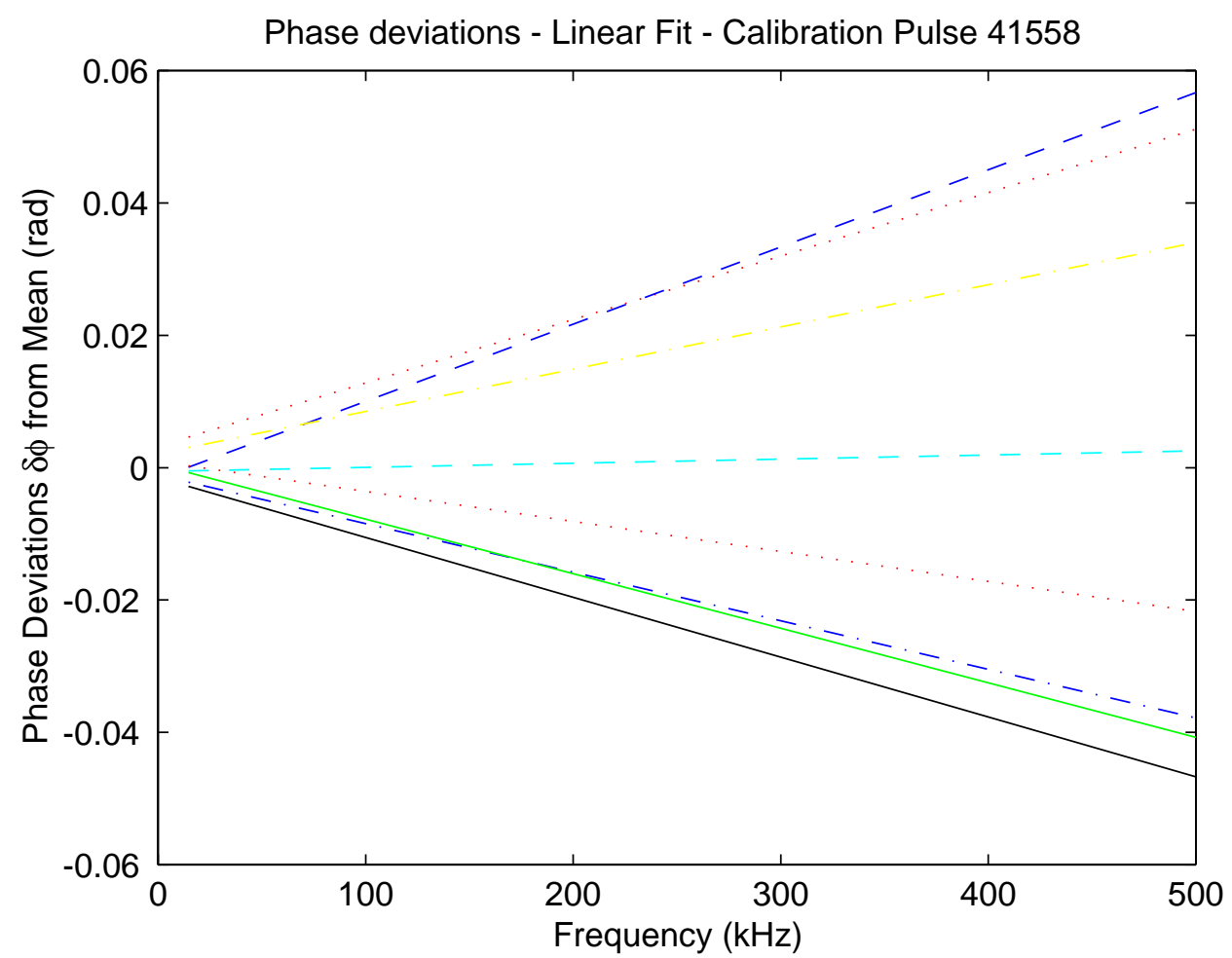

FIG. 4. Phase Response (deviations from mean) of Amplifier-Digitizer Section vs. Frequency.

\section{Calibration Theory for Probe-and-Cable Section}

The transfer function $H_{p}$ for the probe and cable could not be measured directly. Instead a remote technique was developed that relied upon our ability to measure the complex impedance of the probe-and-cable circuit as a function of frequency. A model of the probe-and-cable circuit incorporating the known circuit elements then allows the transfer function to be determined from a parametrized fit to the impedance.

\section{Circuit Model}

The JET Mirnov probe circuits consist simply of the probe itself plus a long $(\sim 100 \mathrm{~m})$ screened twisted-pair cable that brings the signal from the torus hall, through various connections, into the diagnostic hall. The probe has a characteristic impedance due to its resistance and inductance (in series), namely $Z_{p}(s) \equiv R_{p}+s L_{p}$, where $R_{p} \equiv Z(s=0) \approx 50 \Omega$, and $L_{p} \approx 50 \mu H$.

The simplest circuit model which might be reasonable accounts for the probe's inductance and resistance, but neglects the cable except for its capacitance and admittance (resistance), which is in parallel with the probe as shown in the top of Fig. 5. A model using conventional circuit elements is feasible because the wavelength of a 500-kHz signal (at the Nyquist frequency for the digitizers) is $600 \mathrm{~m}$, whereas the size of the probe-and-cable system is about 100 m. However, we knew from preliminary impedance measurements that this single-pole model would be inadequate. The model which we originally sought to use for the JET probes also accounts for the cable's inductance and (for completeness) resistance, as shown in the middle of Fig. 5. This proved to be adequate only for low frequencies at JET. One possible reason is that the probe cables make connections (at the probe, at the limb junction inside the torus hall, and so on), and the different sections of cable may have slightly different physical characteristics. Consequently the work done here involves the physics of the generalized k-th order circuit shown in the bottom of Fig. 5. In all cases the measurement device at the output (in our case, the $\mathrm{KC1F}$ digitizer input) is presumed to have a high impedance

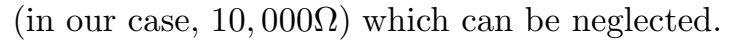



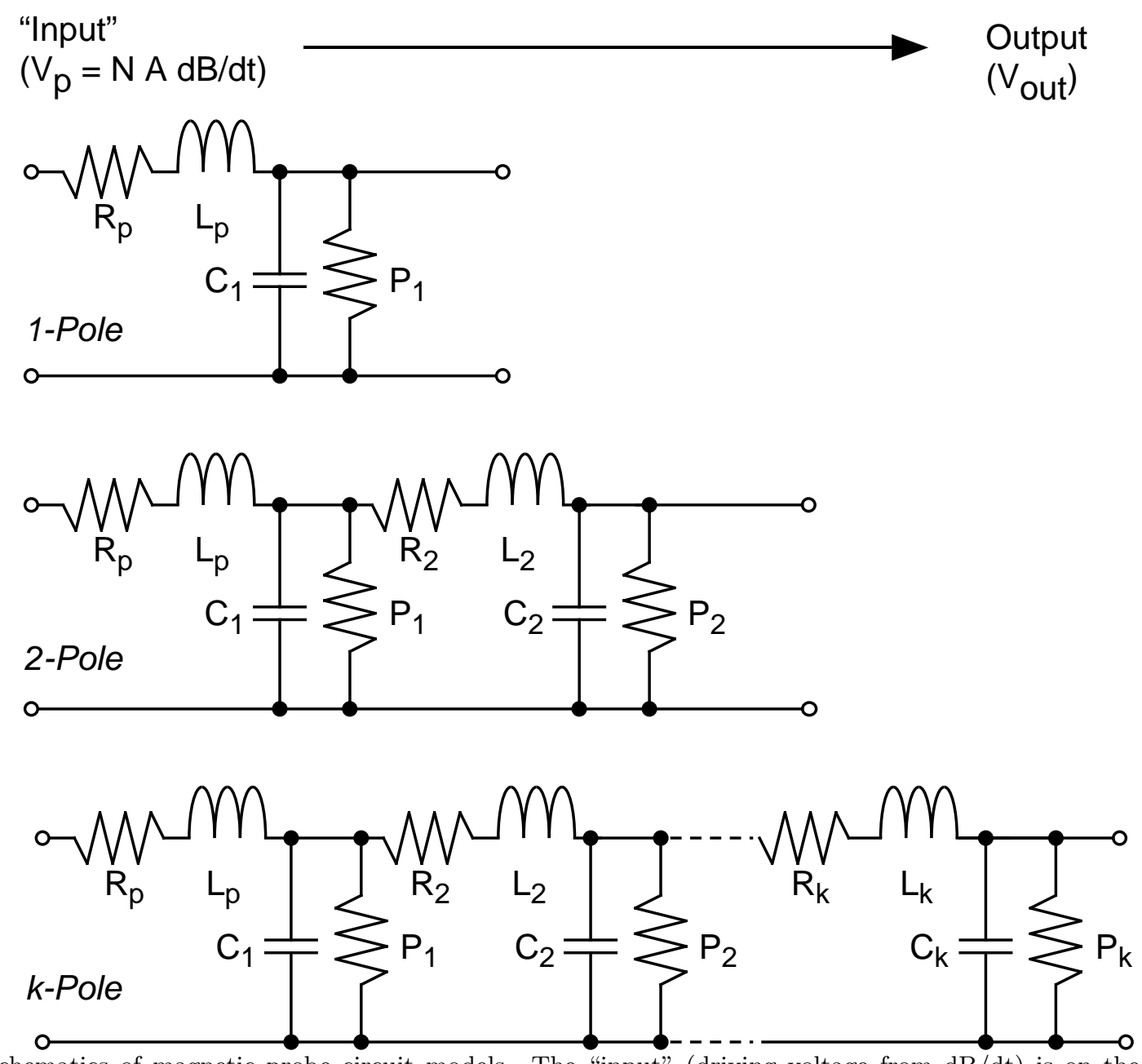

FIG. 5. Schematics of magnetic probe circuit models. The "input" (driving voltage from dB/dt) is on the left, and the "output" (which goes to the amplifier-digitizer system) is on the right. The "P" terms are for the admittances (parallel resistances, $\mathrm{P}=1 / \mathrm{R}$ ) of the transmission cable. Top: simple single-pole model (model A) which includes the probe and one set of elements for the effects of the cable. Middle: second-order model (model B) which has 2 sets of parameters for the cable. Bottom: generalization of the circuit model to a k-pole system with an arbitrary number of parameters (model K).

\section{Impedance of Model Circuit}

For the simple 1-pole circuit (Model A), the impedance is easily determined to be

$$
Z_{A}=\frac{1}{P_{1}+i \omega C_{1}+\frac{1}{R_{p}+i \omega L p}}
$$

or, in a form that will be more useful later, using $s=i \omega$,

$$
Z_{A}=\frac{R_{p}+s L p}{1+\left(P_{1}+s C_{1}\right)\left(R_{p}+s L p\right)}=\frac{N_{A}}{D_{A}},
$$

where $N_{A}$ and $D_{A}$ are polynomials in s representing the numerator and denominator of the impedance.

The 2-pole circuit (Model B) just consists of the 1-pole circuit, plus $R_{2}$ and $L_{2}$ in series and $C_{2}$ and $P_{2}$ in parallel, so the impedance becomes

$$
Z_{B}=\frac{1}{P_{2}+s C_{2}+\frac{1}{R_{2}+s L 2+Z_{A}}},
$$

Substituting $Z_{A}$ and grinding out the algebra is not particularly informative for our purposes. It is clear, though, that we are dealing with rational functions in s. Also, the order of the numerator and denominator polynomials increases 
with the number of poles in the circuit, and the number of circuit elements is 4 times the order of the circuit. Looking to the $\mathrm{k}$-th order circuit, it should be clear that as the order of the circuit increases the algebra becomes increasingly unmanageable (as was verified by doing the 4th-order circuit by hand!).

However, one can derive recursion rules for how $\mathrm{Z}$ changes as the order of the circuit is increased. We can start with the rational function $Z_{k}=N_{k} / D_{k}$, where $\mathrm{N}$ and $\mathrm{D}$ are the numerator and denominator polynomials. Then in adding another segment, we simply hitch on $R_{k+1}$ and $L_{k+1}$ in series and $C_{k+1}$ and $P_{k+1}$ in parallel. To simplify the notation henceforth, let's define

$$
\begin{aligned}
X_{k+1} & \equiv P_{k+1}+s C_{k+1} \\
Y_{k+1} & \equiv R_{k+1}+s L_{k+1} .
\end{aligned}
$$

$\mathrm{X}$ is now the parallel term and $\mathrm{Y}$ the series term. Note that while $\mathrm{X}$ and $\mathrm{Y}$ are both linear functions in s, Y has units of Ohms and $\mathrm{X}$ has units of $1 /$ Ohms. Then the impedance $\mathrm{Z}$ becomes

$$
Z_{k+1}=\frac{1}{X_{k+1}+\frac{1}{Y_{k+1}+Z_{k}}} .
$$

This can be processed and put into rational form again as

$$
Z_{k+1}=\frac{N_{k}+D_{k} Y_{k+1}}{X_{k+1}\left(N_{k}+D_{k} Y_{k+1}\right)+D_{k}}
$$

By inspection of equation 18 one can identify the recursion rules

$$
\begin{aligned}
N_{k+1} & =N_{k}+D_{k} Y_{k+1} \\
& =\sum_{j=1}^{k+1} D_{j-1} Y_{j} \\
D_{k+1} & =D_{k}+X_{k+1} N_{k+1} \\
& =D_{k}+X_{k+1} \sum_{j=1}^{k+1} D_{j-1} Y_{j}
\end{aligned}
$$

Now things start to get fun. Since the impedance is a rational function, it can be represented by two vectors in the linear space of polynomial functions (one for the numerator and one for the denominator). Furthermore the process of adding series terms $\left(Y_{k}\right)$ and parallel terms $\left(X_{k}\right)$ to a given circuit (and thus transforming $\mathrm{Z}=\mathrm{N} / \mathrm{D}$ via the recursion rules) can be represented by linear operators in this space. The elements of each operator are determined by the circuit elements that are added in each stage.

To distinguish the linear-space representation from the physical circuit description, let us say $N_{k} \equiv \mathbf{B}_{k}$ and $D_{k} \equiv \mathbf{A}_{k}$ in the linear space, where $\mathbf{B}_{k}=\left[b_{k+1}+b_{k} s+\ldots+b_{0} s^{k}\right]$ and $\mathbf{A}_{k}=\left[a_{k+1}+a_{k} s+\ldots+a_{0} s^{k}\right]$ (using MATLAB-style polynomial notation, consistent with the code written to implement this system).

The process of polynomial multiplication is the same as that of convoluting the vectors whose elements are the polynomial coefficients, so the recursion rules can be rewritten using the convolution matrices. Adding on an inductorresistor pair $Y_{j}=R_{j}+L_{j} s$ in series changes only the numerator of the impedance, with

$$
\mathbf{B}_{\mathbf{j}+\mathbf{1}}=\mathbf{B}_{\mathbf{j}}+\mathbf{L}_{\mathbf{j}} \mathbf{A}_{\mathbf{j}}
$$

where the operator $\mathbf{L}_{\mathbf{j}}$ is now

$$
\mathbf{L}_{\mathbf{j}}=\left[\begin{array}{ccccc}
R_{j} & 0 & 0 & 0 & \cdots \\
L_{j} & R_{j} & 0 & 0 & \cdots \\
0 & L_{j} & R_{j} & 0 & \cdots \\
0 & 0 & L_{j} & R_{j} & \cdots \\
\cdots & \cdots & \cdots & \cdots & \cdots
\end{array}\right]
$$

Adding a parallel component $X_{j}=P_{j}+C_{j} s$ has a satisfyingly symmetric effect,

$$
\mathbf{A}_{\mathbf{j}+\mathbf{1}}=\mathbf{A}_{\mathbf{j}}+\mathbf{C}_{\mathbf{j}} \mathbf{B}_{\mathbf{j}}
$$

where $\mathbf{C}_{\mathbf{j}}$ is, as expected, 


$$
\mathbf{C}_{\mathbf{j}}=\left[\begin{array}{ccccc}
P_{j} & 0 & 0 & 0 & \cdots \\
C_{j} & P_{j} & 0 & 0 & \cdots \\
0 & C_{j} & P_{j} & 0 & \cdots \\
0 & 0 & C_{j} & P_{j} & \cdots \\
\cdots & \cdots & \cdots & \cdots & \cdots
\end{array}\right]
$$

By starting off with the null circuit $\mathbf{B}_{\mathbf{0}}=\mathbf{0}$ and $\mathbf{A}_{\mathbf{0}}=\mathbf{1}$, and then systematically adding circuit elements by applying the appropriate transformations $\mathbf{L}_{\mathbf{j}}$ and $\mathbf{C}_{\mathbf{j}}$, one can rapidly construct the rational impedance for the whole circuit. Because the operators are invertible, given a rational function for the impedance, one can extract the parameters of the circuit elements $L_{j}, R_{j}, C_{j}$ and $P_{j}$. This will prove useful later. Our next step is to examine the relationship between the impedance (which we can measure) and the transfer function (which we want to determine).

\section{Relationship Between Impedance and Transfer Function}

In principle, if one has a measurement of the circuit impedance as a function of frequency, plus a knowledge of the circuit structure (though not necessarily the values of the components), one can extract the transfer function between different parts of the circuit. In this case we are interested in the transfer function $H_{p}=V_{\text {out }} / V_{\text {in }}$. The "hard way" to determine $H_{p}$ is to use the inverses of the circuit transformations described above to determine all of the component values from a polynomial fit to the impedance, and then construct the transfer function from the known circuit structure. This is not a simple problem and is likely to be fraught with numerical error if the impedance measurement or fit is imperfect, or if certain circuit elements have small impedances.

A much more direct method is available for the class of circuits of interest here, because there is a surprisingly simple and direct relationship between the rationalized impedance $Z_{k}(s)=N_{k}(s) / D_{k}(s)$ and $H_{k}$ :

Theorem 1 For circuits of the form in Fig. 5 of any order $k, H_{k}=1 / D_{k}$.

In other words, if the impedance $\mathrm{Z}$ is represented as a rational function in $\mathrm{s}, \mathrm{Z}=\mathrm{N} / \mathrm{D}$, then the transfer function $\mathrm{H}$ from input to output is always $1 / \mathrm{D}$.

This theorem is fundamental to the calibration technique because it implies that one can simply measure the impedance, find the lowest-order rational function that fits it, cast it into appropriate form, and then use the denominator as the transfer function, with no additional work. The proof of the theorem is given in Appendix A 2.

\section{Rational Function Fit to the Impedance}

The basic theory for the calibration of the probe-and-cable section is now assembled. We will measure the impedance as a function of frequency, fit this to a suitable rational function, and then use the denominator for the corresponding transfer function. Furthermore we can verify the rational fit by taking the fitted impedance and extracting the values of the parameters of the circuit (within the limits of numerical error) to see if they are reasonable.

In the Appendix, Section A 3 provides complete technical information on the benchmarking and validation of this method and its numerical implementation, using a probe-like test circuit with known structure and component values. Here we will proceed to apply the calibration method to the JET Mirnov probes themselves. Later sections will assemble the entire transfer function and describe how it is applied to calibrate the data.

\section{Calibration Results for the JET Probes}

\section{Impedance Measurements}

The procedure used to measure the probe circuit impedance vs. frequency is the same as for the Test Circuit and is described in Section A 3. The frequency range tested was generally 20 to $1000 \mathrm{kHz}$, using $1.02 \mathrm{~s}$ linear sweeps in 1997, and $10.1 \mathrm{~s} \log$ sweeps in 1998. Although part of the measurement apparatus (S-parameter test set) is nominally calibrated only for frequencies above $100 \mathrm{kHz}$, the Test Circuit results give confidence that the impedance measurements are acceptable down to $50 \mathrm{kHz}$ or so. Reproducibility checks in 1998 found sweep-to-sweep changes of $1 \%$ or less in the impedance amplitude and phase, so while there may be systematic errors there appears to be little random error in the impedance measurements. Altogether 9 sets of measurements were made from March 1997 through March 1999. 
As a DC (zero-frequency) check, the overall probe circuit resistance was measured with a multimeter. Except for the probes which were not functioning, the measured resistances ranged from $49.7 \Omega$ to $52.1 \Omega$, quite close to the nominal value of $50 \Omega$. These results were consistent with the extrapolation of the $\mathrm{Z}$ measurements from $20 \mathrm{kHz}$ down to 0 $\mathrm{kHz}$.

Fig. 6 shows the standard impedance measurements for the 3-probe high-resolution toroidal array, and Fig. 7 shows the measurements for 4 of the 10 probes in the main toroidal set. Note the strong similarities within each set and the marked differences between the two sets.
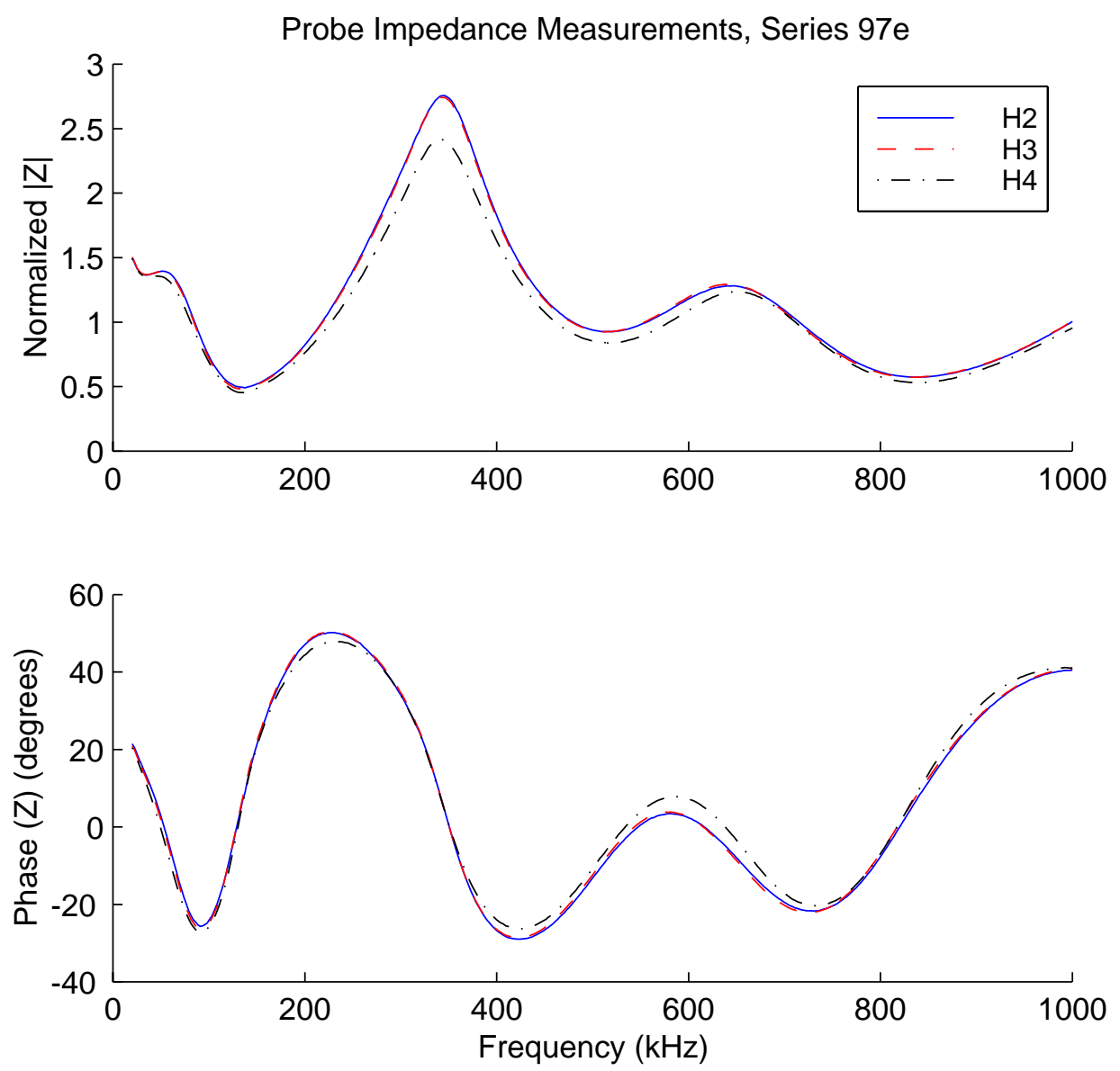

FIG. 6. Impedances of high-resolution JET toroidal probe array circuits, 1997/03/10 data. Probes H302, H303, and H304. 

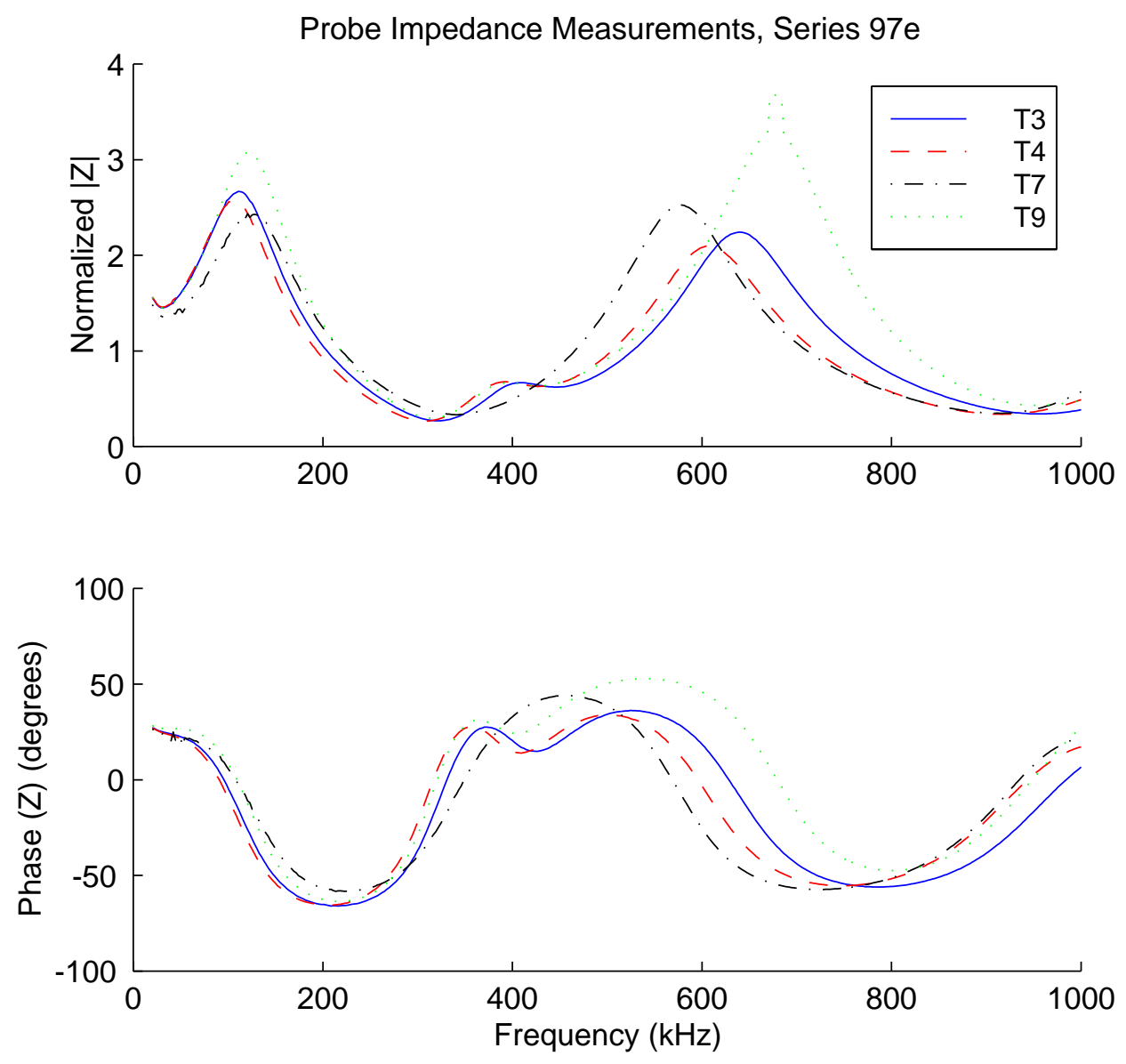

FIG. 7. Impedances of principal JET toroidal probe circuits, 1997/03/10 data. Probes T3, T4, T7, and T9 are shown.

\section{Rational Fits to the Impedances}

The data was restricted to the range $0-700 \mathrm{kHz}$ and then a series of rational functions of increasing order were fit to each of the impedance measurements, until a good fit was obtained. For all the probes except T7 the first reasonable fit was obtained with a seventh-order numerator and sixth-order denominator, corresponding to a probe circuit with 4 series and 3 parallel sections. For T7 a 5/4 fit worked fine. Fig. 8 shows a typical result. The RMS fit errors in this case were $\delta|Z|_{r m s}=0.989 \Omega$ for the amplitude and $\delta \phi_{R M S}=0.8437^{\circ}$ for the phase. The range for all the probes discussed here $(\mathrm{T} 3, \mathrm{~T} 4, \mathrm{~T} 7, \mathrm{~T} 9, \mathrm{H} 302, \mathrm{H} 303, \mathrm{H} 304)$ was $0.674 \leq \delta|Z|_{r m s} \leq 1.288 \Omega$ and $0.773^{\circ} \leq \delta \phi_{R M S} \leq 0.874^{\circ}$. 

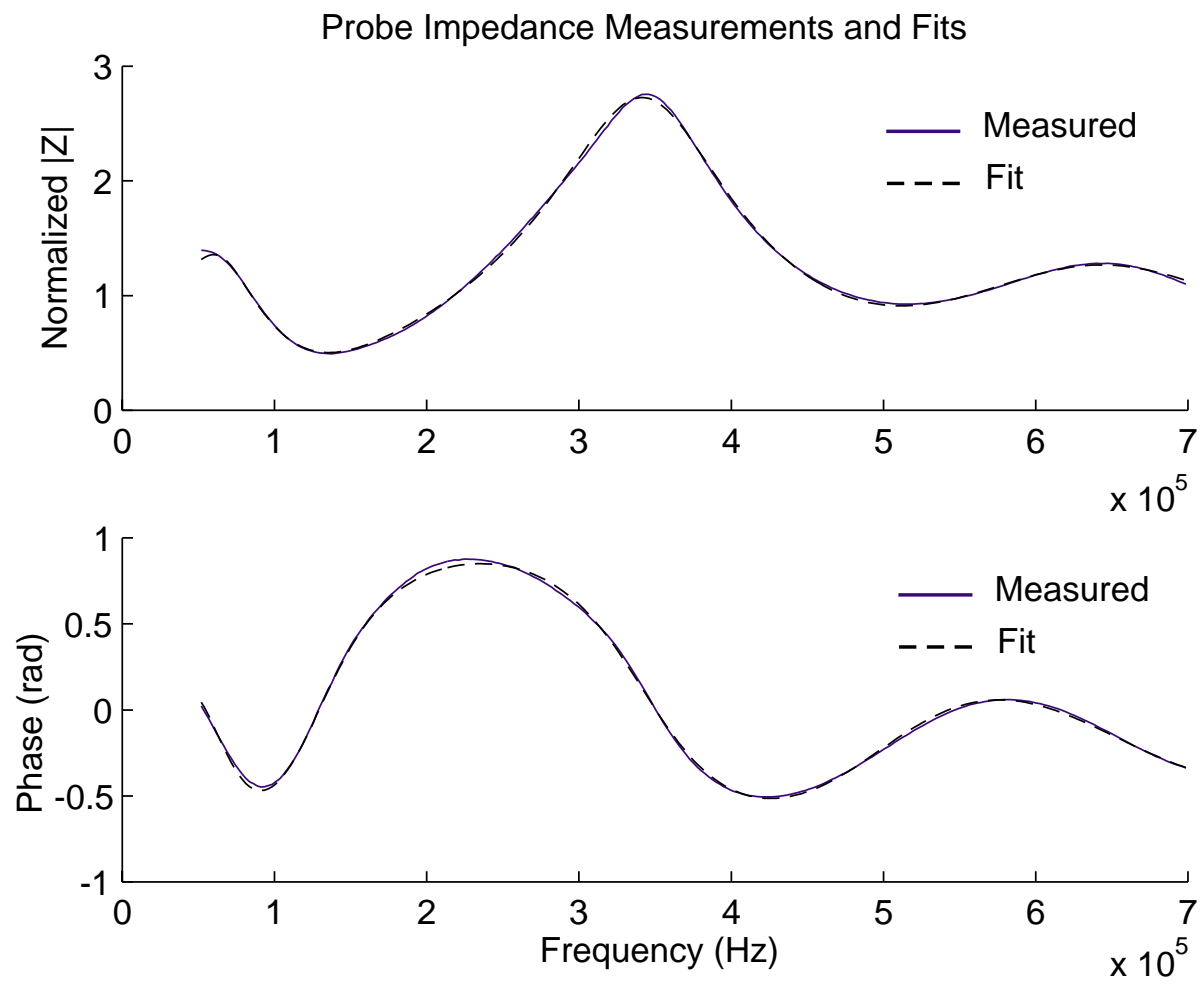

FIG. 8. Sample impedance fit showing results for JET probe H302.

Once the fits were obtained, the transfer function was recorded from the denominator polynomial coefficients, along with the fit error information.

\section{Before-and-After Comparison}

A before-and-after comparison was done to verify that the probe characteristics did not drift over the course of the campaign. No detectable changes were found in any of the probe impedances from March 1997 through January 1998. However, in February 1999 the impedance data for probe T7 showed extensive noise at $50 \mathrm{kHz}$, so this probe was disconnected and that channel was connected to T9, which also turns out to have the highest overall sensitivity. Fig. 9 shows one example. 

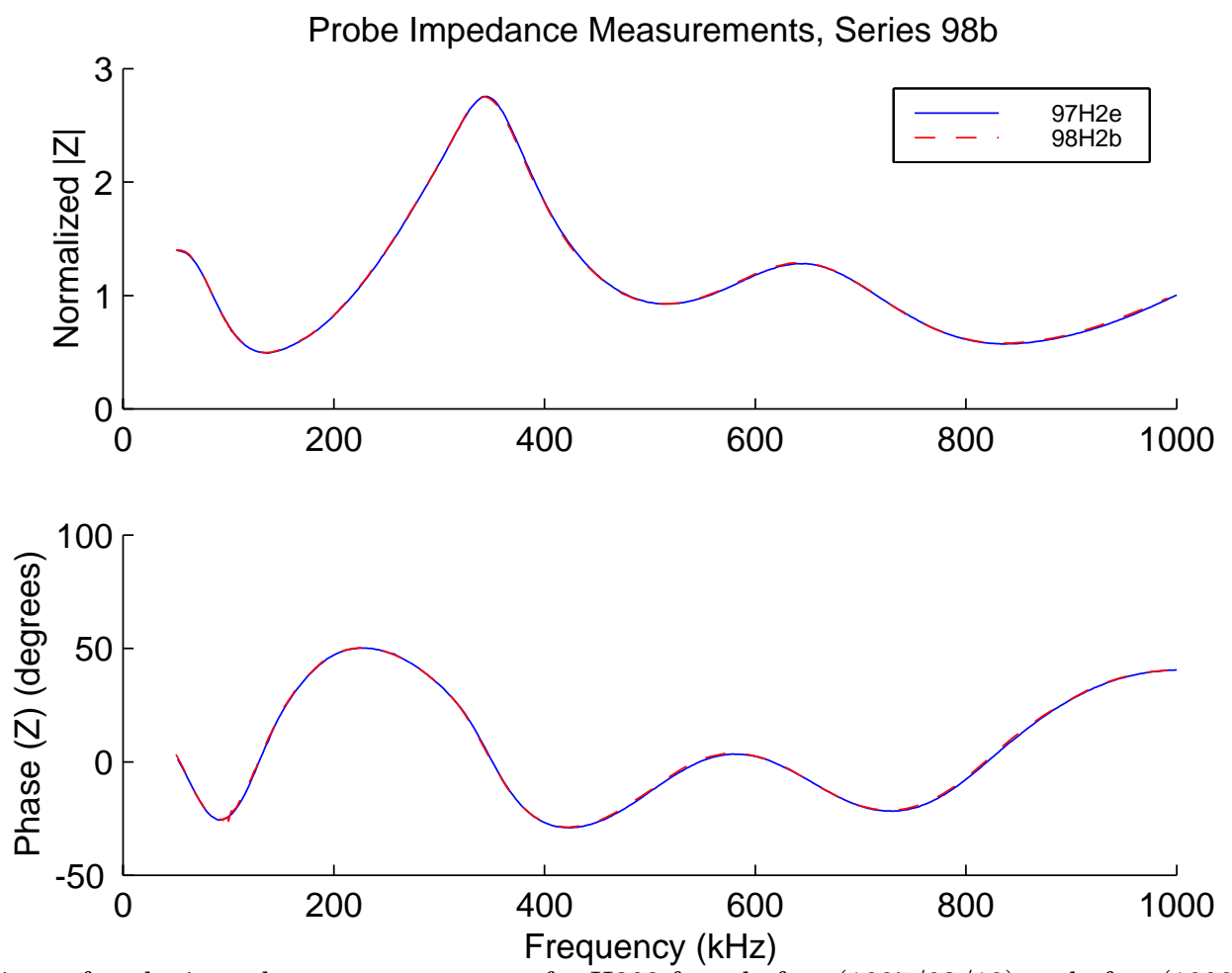

FIG. 9. Comparison of probe impedance measurements for H302 from before (1997/03/10) and after (1998/01/18) the DTE1 experimental campaign.

\section{Effects of Changing Vacuum Vessel Temperature}

JET normally operates at a vacuum vessel (probe) temperature of $320^{\circ} \mathrm{C}$, but a few experiments carried out in late 1997/early 1998 used a reduced temperature of $150^{\circ} \mathrm{C}$. The lower temperature can affect the plasma-wall interactions and is of interest for future fusion reactors. The reduced wall temperature also affects the probe impedance, as shown in Fig. 10. This is presumably due to minor changes in the probe resistance with temperature and in the probe inductance with thermal expansion. The change in the impedance is significant enough to conclude that a complete recalibration of all the probes is necessary if reliable results (particularly mode numbers) are needed at wall temperatures other than $320^{\circ} \mathrm{C}$. 

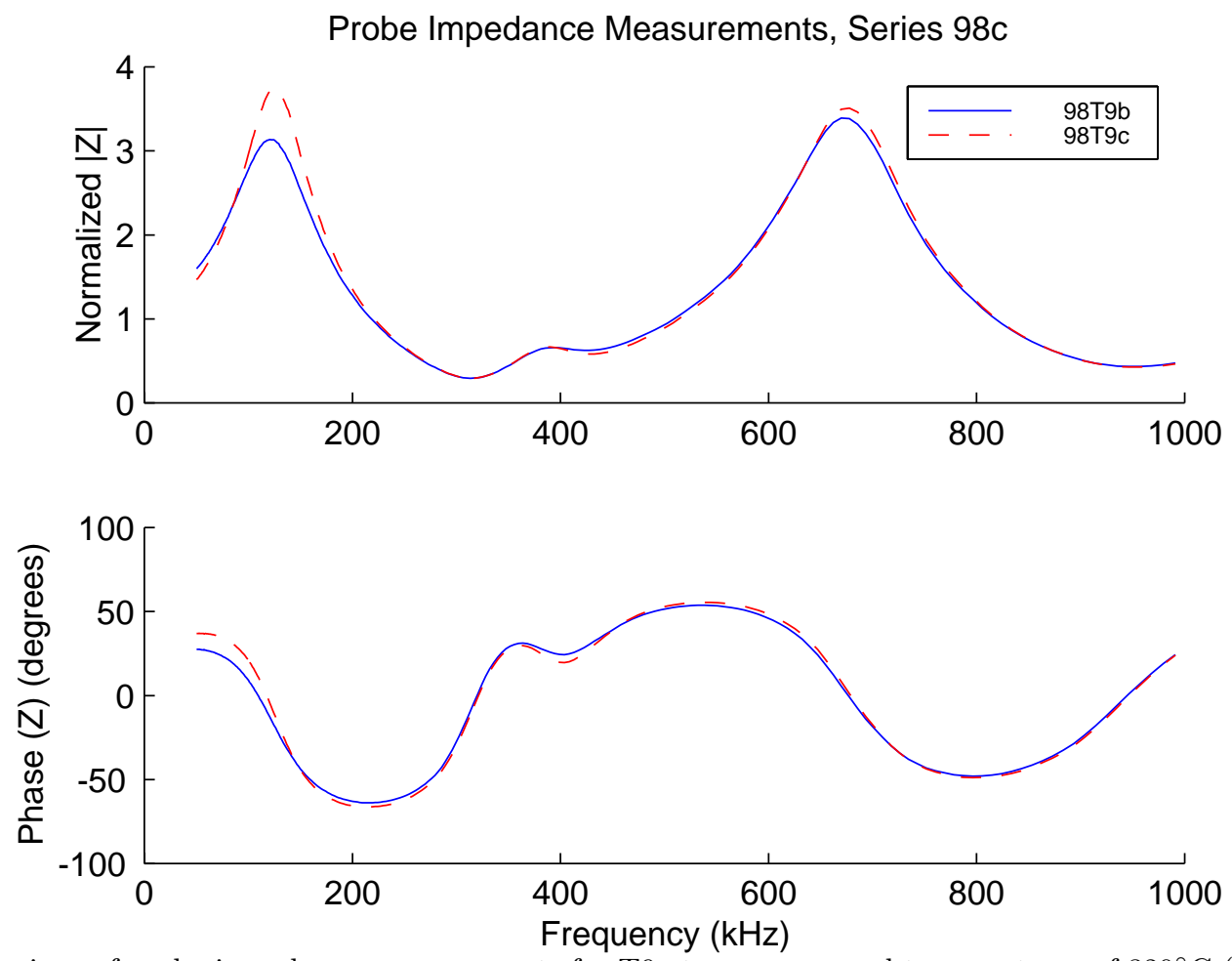

FIG. 10. Comparison of probe impedance measurements for T9 at vacuum vessel temperatures of $320^{\circ} \mathrm{C}(1998 / 01 / 18$, series b) and $150^{\circ} \mathrm{C}(1998 / 02 / 02$, series c).

\section{E. Overall Calibrated Transfer Function}

The overall transfer function is determined by taking the product of the transfer functions $H_{a}$ (counts/volt), $H_{p}\left(V_{\text {out }} / V_{\text {in }}\right)$, and $V_{\text {in }} / \delta B=N A s$ for the amplifier-digitizer section, probe-and-cable section, and probe head, respectively. This rational function is then inverted to extract the magnetic fluctuation signal $\delta B(s)$ in Tesla/count from the digitized data. The only remaining calibration issue is how to apply the frequency-domain transfer function to calibrate the time-domain data. Section IV details the solution that was adopted, its implementation in the data-processing software, and the resulting overall calibration curves.

\section{DATA PROCESSING}

Here we first review the data pipeline hardware and performance, then explain how the calibration results from Section III are applied to the data, and summarize the data processing capabilities that have been implemented. A graphical overview of the entire system is provided by Fig. 1. Additional reference information on the data-analysis software is available in Appendix D of the author's Ph.D. dissertation. ${ }^{5}$.

\section{A. Data Pipeline}

As explained in Section III, the JET fast magnetics system "KC1F" uses a toroidal array of magnetic pickup coils (Mirnov probes) hooked up to a fast-sampling analog-to-digital converter (ADC) connected to a PC which is linked into the UNIX-based JET COntrol and Data Acquisition System (CODAS) through the Windows-NT DATANET network. The PC serves as the local acquisition control system and provides temporary data storage.

The $\mathrm{KC1F}$ system is triggered remotely by CODAS at a time specified by the diagnosticians in the JET control room. After the data collection, the data is piped from the ADCs through the PC and onto the JET DATANET server. The server supplies a data storage area on hard disk of upwards of $2 \mathrm{~GB}$, allowing data to be accumulated throughout the day. At the end of the session (or by special request), the data is finally collected by CODAS and 
transferred to the IBM 3090 mainframe system, which has a large data silo for permanent storage. A schematic of this data pipeline is shown in Fig. 11.

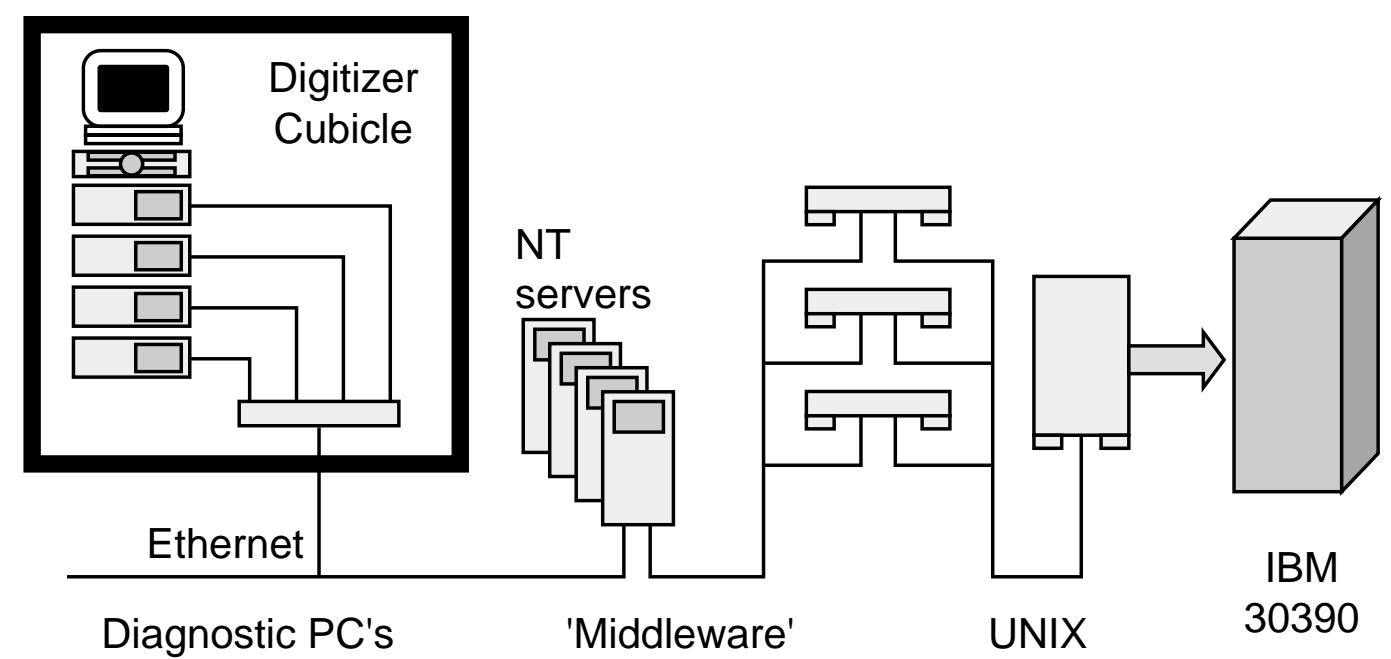

FIG. 11. Schematic of JET Data Acquisition Pipeline. Adapted from figure JG98828c courtesy JET Graphics Office.

\section{Pipeline Performance}

The $\mathrm{KC1F}$ system acquires a large volume of data by JET standards (over 1/10 of the total data each pulse), and the data pipeline is complicated. Furthermore JET operates routinely, taking roughly 30 pulses per day, 5-6 days per week, for several months at a time, resulting in a total of over 4000 pulses/year, and KC1F generally acquires data for every pulse. The transient recorder takes only 4 seconds to record the data in real time, but the ratio of the pipeline bandwidth to the data file size is small (much like a small snake trying to swallow a large cow), so the data archiving takes many minutes. The performance of the data pipeline therefore became an important issue which influenced the data analysis techniques.

Once acquired at the transient recorder, the 32 million samples of 12-bit ADC data (totalling 64 megabytes) are transferred onto the hard disk of the controlling PC. This takes about 5 minutes. The PC must unfortunately swap the byte order of each ADC data point, in order to make it compatible with the integer data format of the archiving system; this takes another 5 minutes. The reordered data is then transferred as a single 64-MB file over the local Windows NT network to a server PC, in another 5 minutes or so. At this point the data is ready to be picked up by the central data acquisition computers, which transfer the data to a Sun UNIX system and then to the data silo attached to the IBM 3090 mainframe which serves as the central data warehouse at JET. This process takes an additional 10-15 minutes.

The entire process takes 25-30 minutes, so it can be carried out between JET pulses provided the interval between shots is long enough. However, this criterion is not routinely met, so the data is usually accumulated on the server PC during the day, and then archived overnight. A storage area of 2 GB (later 2.6) was obtained to allow 30 pulses (later 40) of $64 \mathrm{MB}$ each to be acquired on a daily basis. The total data collection for the 1997-1998 D-T campaign exceeded $200 \mathrm{~GB}$, representing something like $10 \%$ of the data ever archived at $\mathrm{JET}^{38}$. Occasionally the data pipeline will fail, leaving data stranded on the diagnostic PC, so the system was monitored on a daily to weekly basis to prevent the local storage from becoming clogged with old data.

\section{Data Processing Hardware Systems}

Once on the IBM mainframe, the data is available for analysis. Using the mainframe itself for the data processing would consume too much CPU time and cause problems for the rest of the JET operations, so instead the data is transferred on a by-request-only basis to one or more analysis computers. These have included UNIX systems and Macintosh PCs. A set of standardized and automated software tools was written in MATLAB to allow data to be obtained from the archive and standard analyses to be performed on both UNIX and Mac systems. The physics of these standard analyses are described below, following a brief description of certain aspects of the computer hardware. 
We first began analyzing the data in the spring of 1997 using a Sparc 2+ UNIX workstation (gen-off-2) with 500 MB of disk space. The disk space proved limited considering the size of the data files, and it was also rather difficult to generate and print high-quality figures for presentations and publications. so new hardware was sought.

A second copy of MATLAB was installed in the summer of 1997 on a Macintosh PowerBook 5300c with 64 MB RAM and 1.8 GB of disk space. This was somewhat slower than the Sparc, but was useful for code development, data storage, and production of figures, and allowed the Sparc to be used solely for computationally intensive analysis jobs. The MATLAB software and data-importing tools were upgraded to run on both the Mac and UNIX systems. The disk space was increased by adding a 100 MB removable disk (Zip) drive, and later a 1-GB removable disk (Jaz) drive. The 1-GB cartridges proved expensive, so the next solution was a CD-ROM recorder which could archive data and figures to cheap 650 -MB compact digital disks.

In early 1998 the Mac system was upgraded to a Macintosh PowerBook G3 (250 MHz CPU) with $160 \mathrm{MB}$ of RAM and a 4.5 GB hard disk. This improved the data transfer and processing rates considerably over the Sparc, while supplying additional storage and maintaining compatibility with the external drives used with the previous Macintosh system. It became possible to carry out full mode-number analyses (see below) for a megasample of data at a time.

Planning began in mid-1998 to migrate back to a purely UNIX-based system which could be handed over to existing JET staff at the conclusion of the project. The final computer which arrived in April 1999 is a PC in the JAC cluster running the Linux operating system. The JAC cluster has direct access to the IBM data, which speeds up the analysis considerably, though the CPU is not particularly faster than the Macintosh PowerBook G3. The new system has $384 \mathrm{MB}$ of RAM and $16 \mathrm{~GB}$ of disk space, allowing full spectrogram and mode-number analyses to be carried out routinely for the first time.

\section{B. Signal Calibration via Digital Filtering}

As explained in Section III, the overall transfer function calibration can be obtained as a rational function in frequency (counts/Tesla). This rational function can then be inverted (Tesla/count) to extract the magnetic fluctuation signal $\delta B(s)$ in Tesla from the digitized data in counts. The question then arises how one should apply the frequency-domain transfer function to calibrate the time-domain data.

One possibility would be to construct AC filter circuits to pre-calibrate the data before it is actually digitized. This approach suffers from two main difficulties. First, the complexity of the transfer functions suggests that a relatively complex AC circuit would be needed; the implementation of multiple unique AC filters for all the probes of interest to $\mathrm{KC} 1 \mathrm{~F}$ could become a time-consuming and expensive proposition. Second, if any of the probe characteristics should change (different vacuum vessel temperature, degradation of a connection, etc.), then any data collected prior to the detection of the change would be permanently miscalibrated, and the filter hardware would need to be modified. Rather than implement the calibration with analog hardware, we chose to use digital signal-processing techniques.

One method of applying the transfer function to the data would be to first FFT some section of the data, and then apply the frequency-space transfer function to the FFT'd signal. However, one might want to do other things with the data than FFT it, and with up to $64 \mathrm{MB}$ of data per pulse, the numerical processing in MATLAB (which would require high-level, custom, interpreted functions) could become very slow, particularly if the FFT windows were heavily overlapped to improve the effective time resolution of the diagnostic.

Instead, software was written in MATLAB so that for each combination of probe and digitizer channel that was used, a digital finite-impulse-response filter could be rapidly and semi-automatically constructed to match the response of the true transfer function over the desired frequency range. Software was then written allowing any data file to be rapidly calibrated by using the built-in low-level MATLAB digital filter function to simply filter the data. This approach allows improvements in the transfer function to be applied retroactively to the data. It also makes it possible to rapidly calibrate both new probes and old probes used in new operating conditions, by simply processing a new set of impedance measurements using the semi-automatic software routines.

It is possible to directly convert the transfer function to a filter using the bilinear transformation, but the resulting filter's response only matches the transfer function for frequencies much less than the Nyquist frequency, and in this case we are interested in data right up to the Nyquist frequency ( $500 \mathrm{kHz}$ ). Rather than use a transformation, therefore, we took a brute-force approach and used existing MATLAB filter-fitting routines to design an FIR filter (typically 40th to 48th order) whose amplitude and phase response matched the inverse transfer function to the desired level of accuracy.

The frequency range of the filter fitting was restricted to $30-460 \mathrm{kHz}$ to avoid instabilities near $0 \mathrm{~Hz}$ and $500 \mathrm{kHz}$, so consequently the data outside those ranges is not well calibrated. It turns out that a 40th-order FIR filter can be fitted to most probe transfer functions with maximum error bars of less than $5 \%$ in magnitude and 0.04 radians (2.3 degrees) in phase. The fit order was chosen so that the phase error in the filter fitting is smaller for most frequencies than 
the error from the rational function fits used to determine the transfer function from the impedance measurements described in Section III. The characteristic fit quality for the construction of one such filter is shown in Fig. 12, and the corresponding error information is shown in Fig. 13.
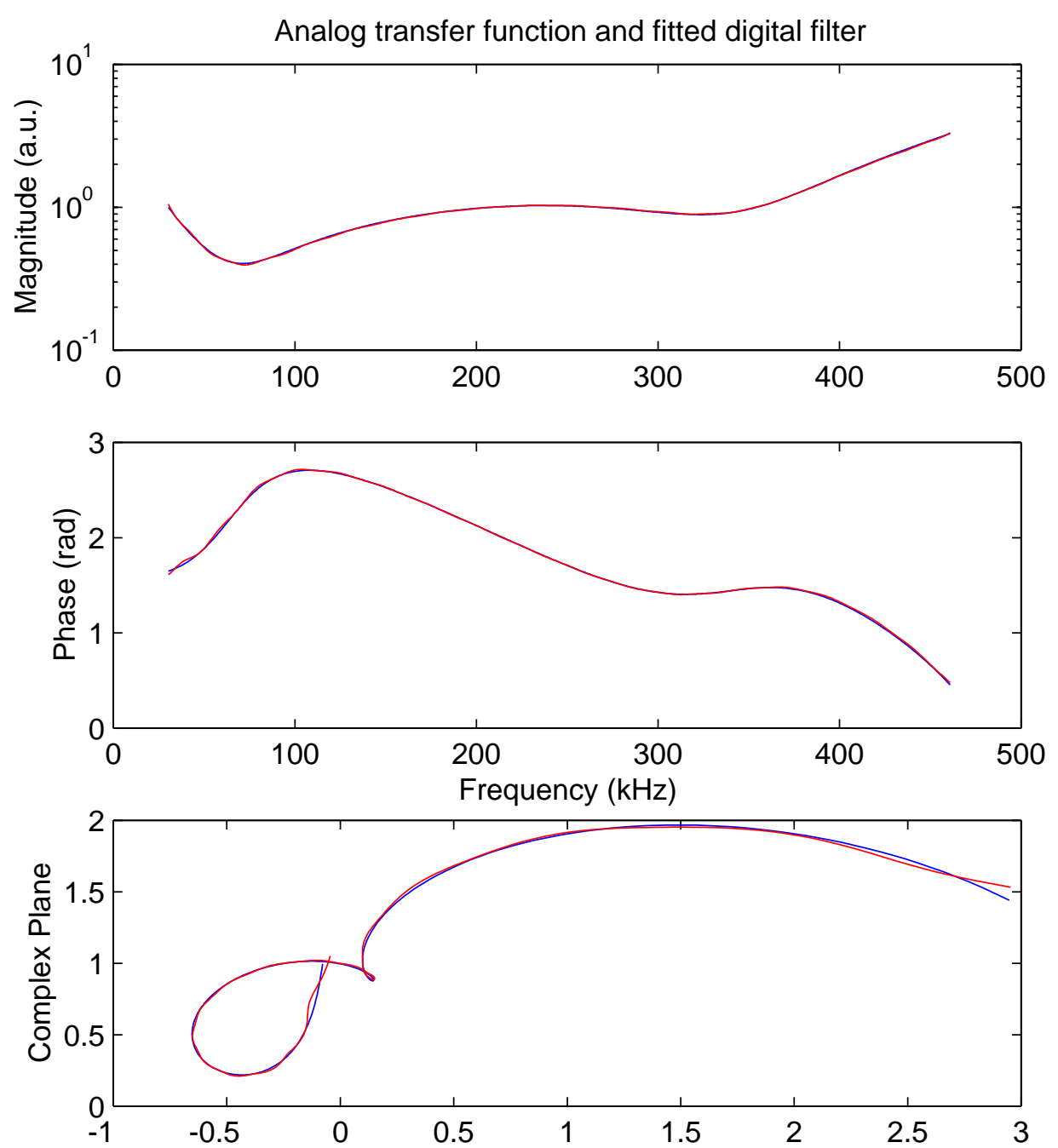

FIG. 12. Sample Comparison of Fitted and Original Transfer Functions. The data is taken from the fitting for Probe H302 connected to KC1F channel 111, valid from pulse 41559 onwards. Top: Amplitude (arbitrarily renormalized). Middle: Phase in radians. Bottom: Complex-plane representation.

The overall known error for the entire calibration process (including the transfer function fit errors and the filter fit error) has also been calculated. For the calibrated region $50-460 \mathrm{kHz}$ the error in the magnitude is typically $3 \%$ or less, with a maximum of $5 \%$. The phase error in the range $50-460 \mathrm{kHz}$ is typically $0.03 \mathrm{rad}$ (1.7 degrees), with a maximum of $0.05 \mathrm{rad}$ (2.9 degrees). 

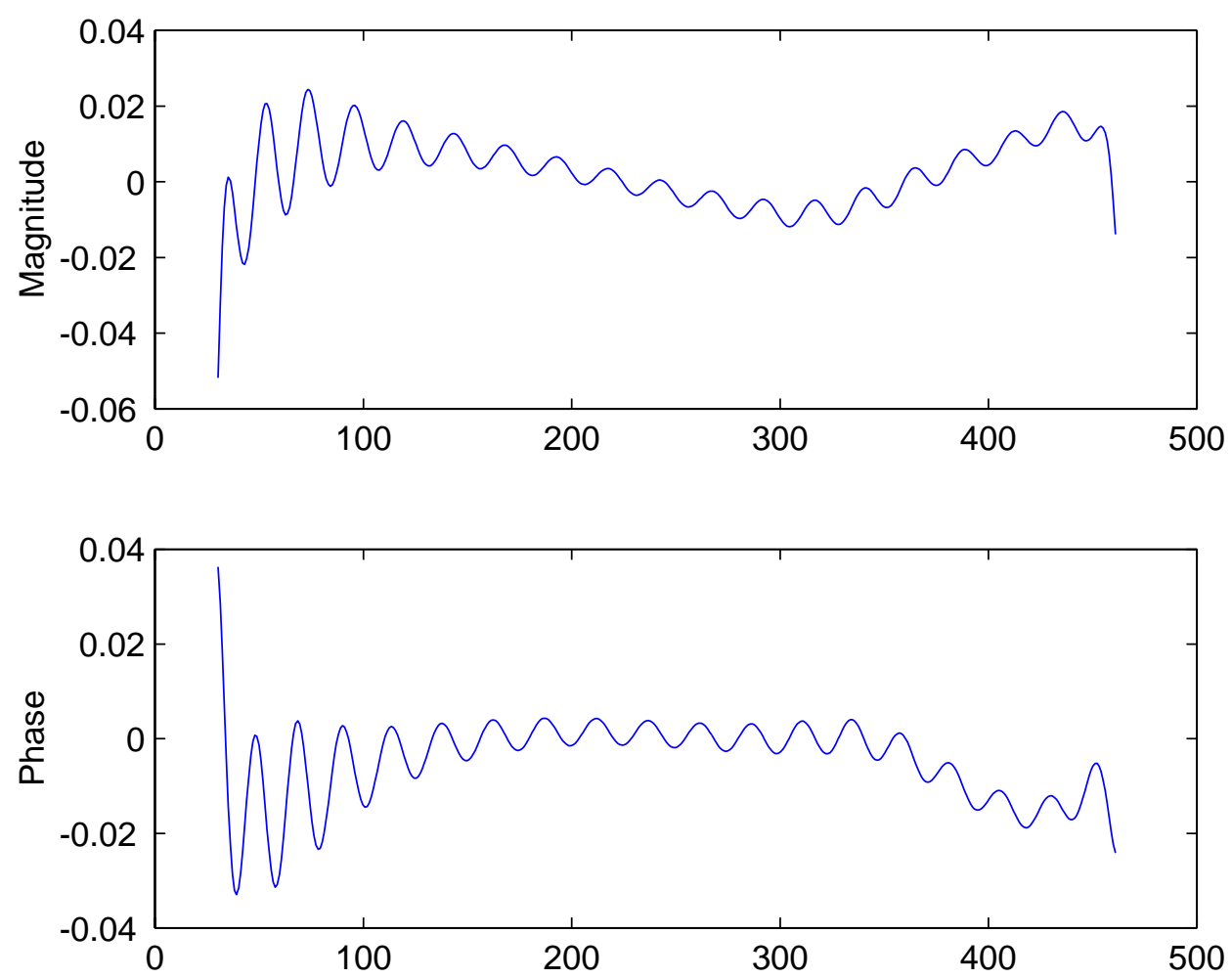

FIG. 13. Transfer Function Filter Fit Errors. Same data as preceding Fig. 12. Top: relative amplitude error vs. frequency in $\mathrm{kHz}$. Bottom: absolute phase error in radians vs. frequency in $\mathrm{kHz}$.

As a cross-check of the filter calibration method, a set of comparisons were done in which the rational transfer function was applied to the FFT of the raw data, and the results compared with the FFT of the calibration-filtered data. As expected the two methods were nearly indistinguishable in their results.

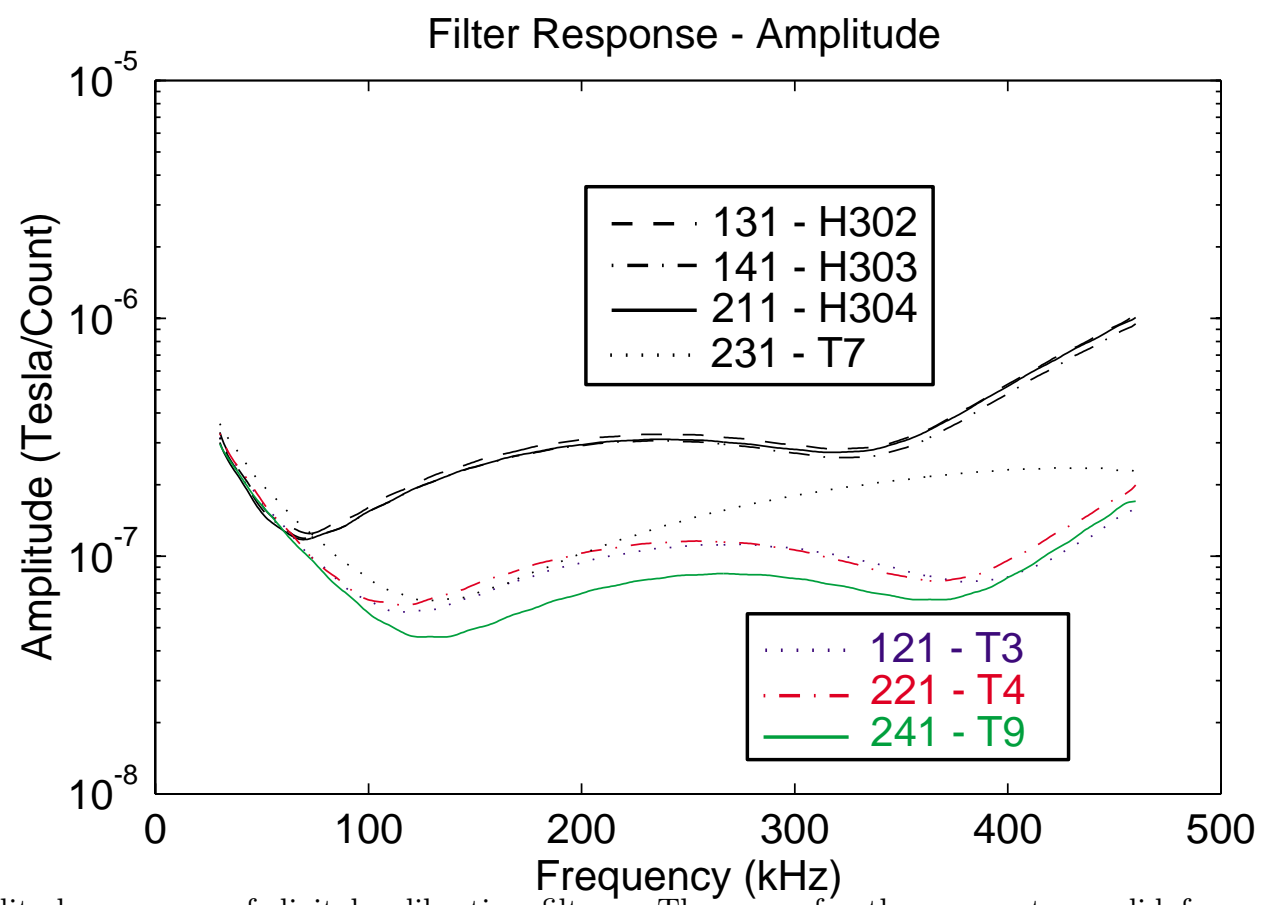

FIG. 14. Amplitude response of digital calibration filters. These are for the parameters valid for pulse \#41723. The three-digit numbers 111, 121, etc. refer to the Sunnyside system input channels; the alphanumeric codes (T1, H302, etc.) indicate the magnetic probe that was connected. 
The overall frequency-domain behavior of the most commonly used calibration filters is shown in Figures 14 and 15. It is tremendously helpful that the calibration curves for the three high-resolution probes (H302, H303, and H304) are nearly identical. This was also evident in the impedance measurements and provides great confidence that these three probes do indeed behave similarly. It is also notable that the calibration indicates that probe T9 has the highest overall sensitivity; this is indeed borne out in the experimental data.

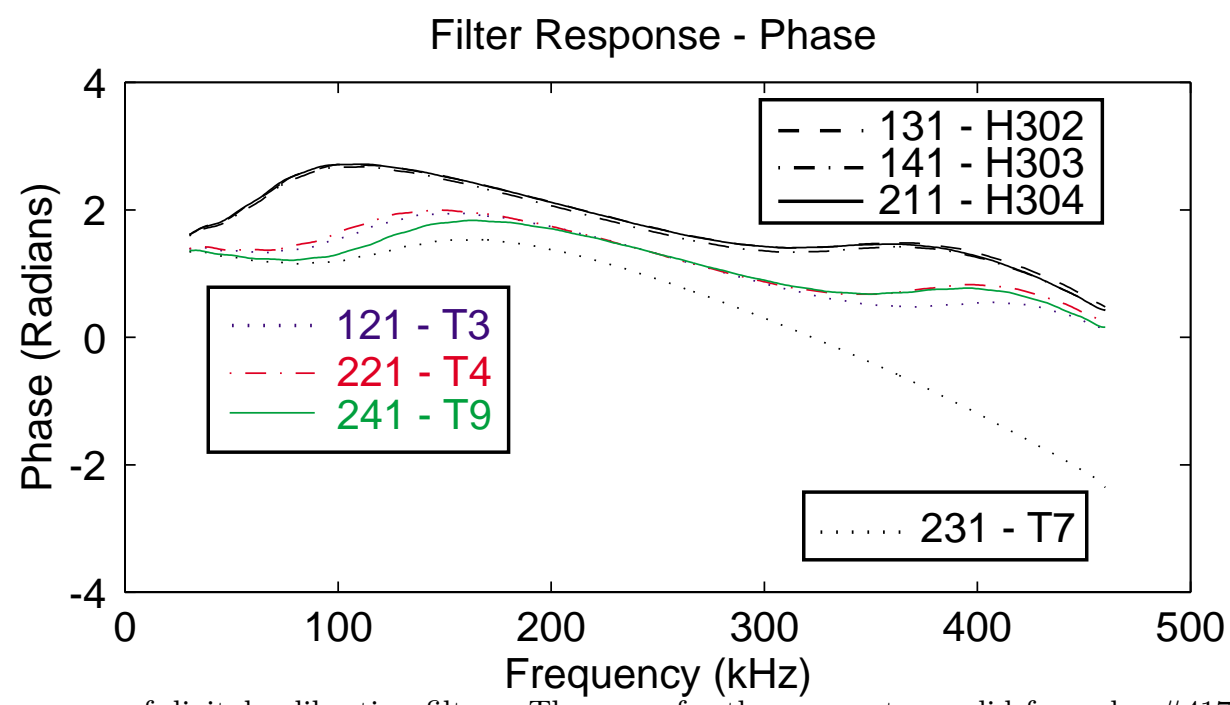

FIG. 15. Phase response of digital calibration filters. These are for the parameters valid for pulse \#41723. The three-digit numbers 111, 121, etc. refer to the Sunnyside system input channels; the alphanumeric codes (T1, H302, etc.) indicate the magnetic probe that was connected.

\section{Data Processing and Analysis Software}

This section describes the capabilities of the data analysis software developed for the $\mathrm{KC} 1 \mathrm{~F}$ system as part of this project. The emphasis is on the scientific decisions and programming principles used in developing the software. Since this software package is now being used by others at JET, a complete user's manual detailing all the software functions was also written by the author. ${ }^{5}$

For processing the JET data MATLAB was again used. ${ }^{46}$ Compared to other high-level tools such as IDL, or lowlevel languages such as $\mathrm{C}$ or FORTRAN, MATLAB had the advantages of being accessible from inside the control room, making use of prior expertise, running with minimal changes on both PC and UNIX systems, and having a high-quality set of built-in library routines for the sort of signal processing that was required. ${ }^{47} \mathrm{~A}$ variety of analysis tools were constructed and assembled in a single menu-driven code ("FastMag"), where they share a common set of software-importing and calibration routines. The code was written in such a way that whenever there is a decision point there is also a default option corresponding to the most likely choice. This method resulted in reasonable flexibility and ease-of-use without requiring a graphical interface.

The fundamental task of the data processing software was to provide useful information on the magnetic fluctuations that were observed. Both the raw digitized signals and filter-calibrated signals could be readily displayed versus time. This basic technique was useful for checking against saturation of the signals by large modes, and also proved essential in studying fast nonlinear phenomena. ${ }^{5}$ ) However, viewing data in the time-domain alone is not very efficient for identifying particular types of modes, and the main focus of the data processing software development was in creating routines to output useful maps of magnetic fluctuation amplitudes and phases versus both time and frequency.

For each pulse and each channel, the calibrated data were time-windowed into groups of $2^{N}$ samples (typically $N=12$; i.e. 4096 points) and then fast-fourier-transformed to give amplitude and phase information as a function of frequency for each window. The data for each time-window were then arranged sequentially to form a 3-D picture of the magnetic fluctuation amplitude as a function of frequency and time. In addition the phase data for the three probes in the high-resolution toroidal array were used to determine the toroidal mode numbers $(n)$ of those coherent fluctuations large enough to have meaningful phase data. The scientific details of the principal functions in the FastMag code are explained below, with sample output. 


\section{Spectrograms}

The most interesting output from the diagnostic is necessarily three-dimensional in nature (amplitude vs. time and frequency), and various techniques were tried in the process of learning how to produce scientifically useful output. Diagnostic development involves both improving the ability to measure quantities of interest and identifying efficient means of identifying phenomena of interest within the data that is measured. This latter becomes especially important when large data sets are involved and one's understanding is time-limited rather than data-limited. It then becomes important to keep in mind that what one is capable of learning from the data depends heavily upon how one goes about looking at it.

It appears from a survey of relevant literature that traditionally the output from fluctuation diagnostics has been displayed as contour plots. These are very efficient at reducing large amounts of data to small numbers of lines highlighting 3-D structure. However, contours can make it difficult to resolve fine structure or to assess amplitudes of small regions. 3-D surface plots are a widely used alternative, and these are much better at resolving fine structure, but they are often difficult to interpret when printed on a 2-D page, and moreover they generally cannot display very much information without becoming cluttered, since one needs 4 intersecting lines to indicate the location of each data "point".

Spectrograms are colormapped images in which every data point is represented by a color indicating its amplitude. These have better overall resolution than contour plots and allow the display of much more information than surface plots, while being nearly as good at resolving fine-grained details, especially since they can be easily zoomed. The use of spectrograms with logarithmic color scales allows modes spanning many orders of magnitude in amplitude to be displayed and identified within a single figure with high resolution in all three dimensions.

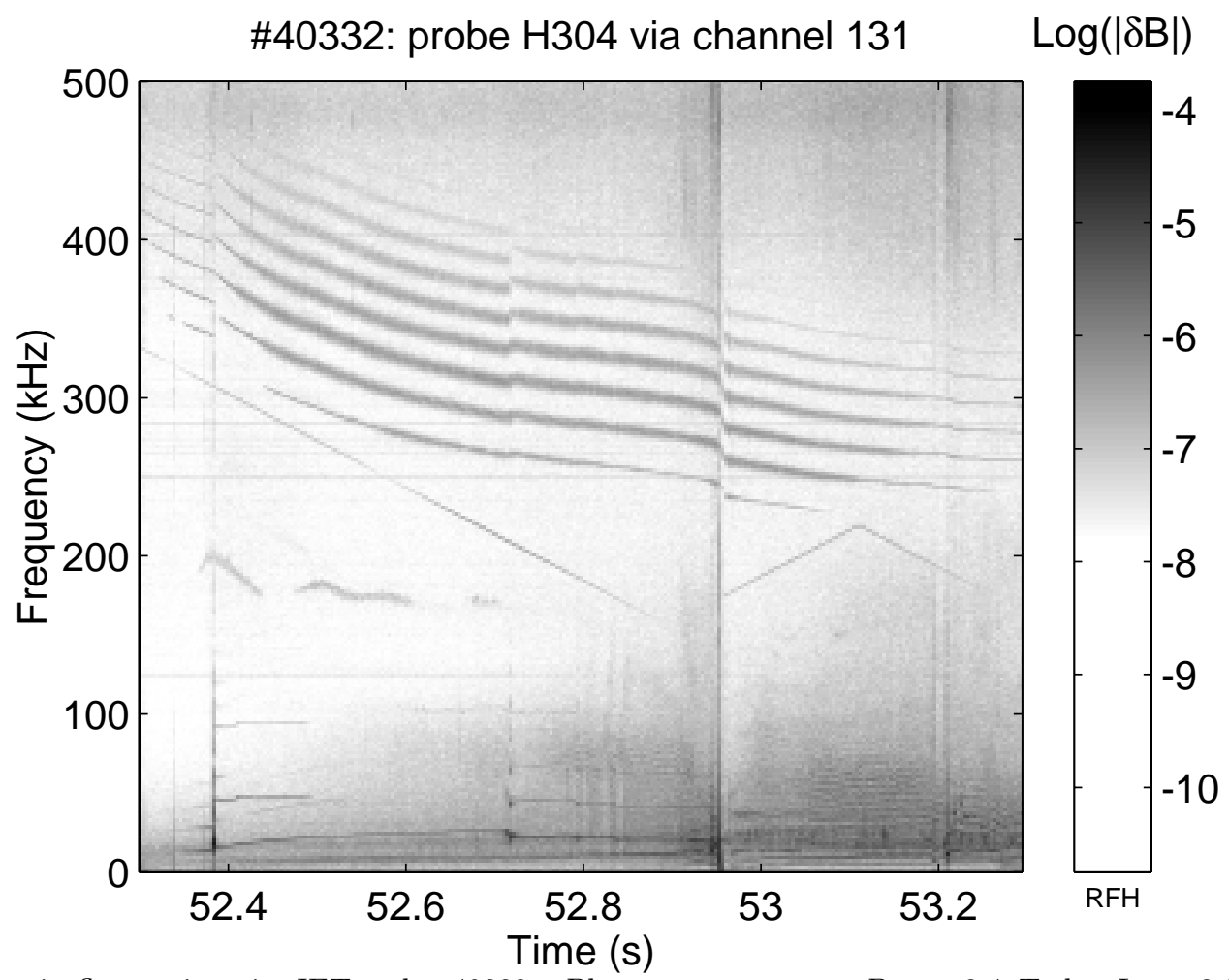

FIG. 16. Magnetic fluctuations in JET pulse 40332. Plasma parameters: $B_{T}=3.4$ Tesla, $I_{p}=3.8$ MA, H-minority $P_{I C R H} \sim 6.5 \mathrm{MW}, P_{N B I} \leq 16 \mathrm{MW}$. Note the Doppler-shifted TAEs across the top $(n=5$ to 12$)$, with line-broadening in the upper left.

Figs. 16 and 17 illustrate the value of spectrograms in identifying one of the most interesting phenomena discovered with KC1F, namely the pitchfork-splitting of the TAEs in JET, in which a single mode appears to split into several submodes at a certain threshold. ${ }^{6}$ The mysterious line-broadening shown Fig. 16 is revealed in the zoomed Fig. 17 to be the splitting of individual TAEs into 3, 5, or 7 separate submodes. The submodes are very near the original mode in frequency space (relative to the resolution of the FFTs), and thus the splitting would not have been as easily seen in a contour plot. Nor could this amount of data have been readily plotted as a 3-D surface plot. 


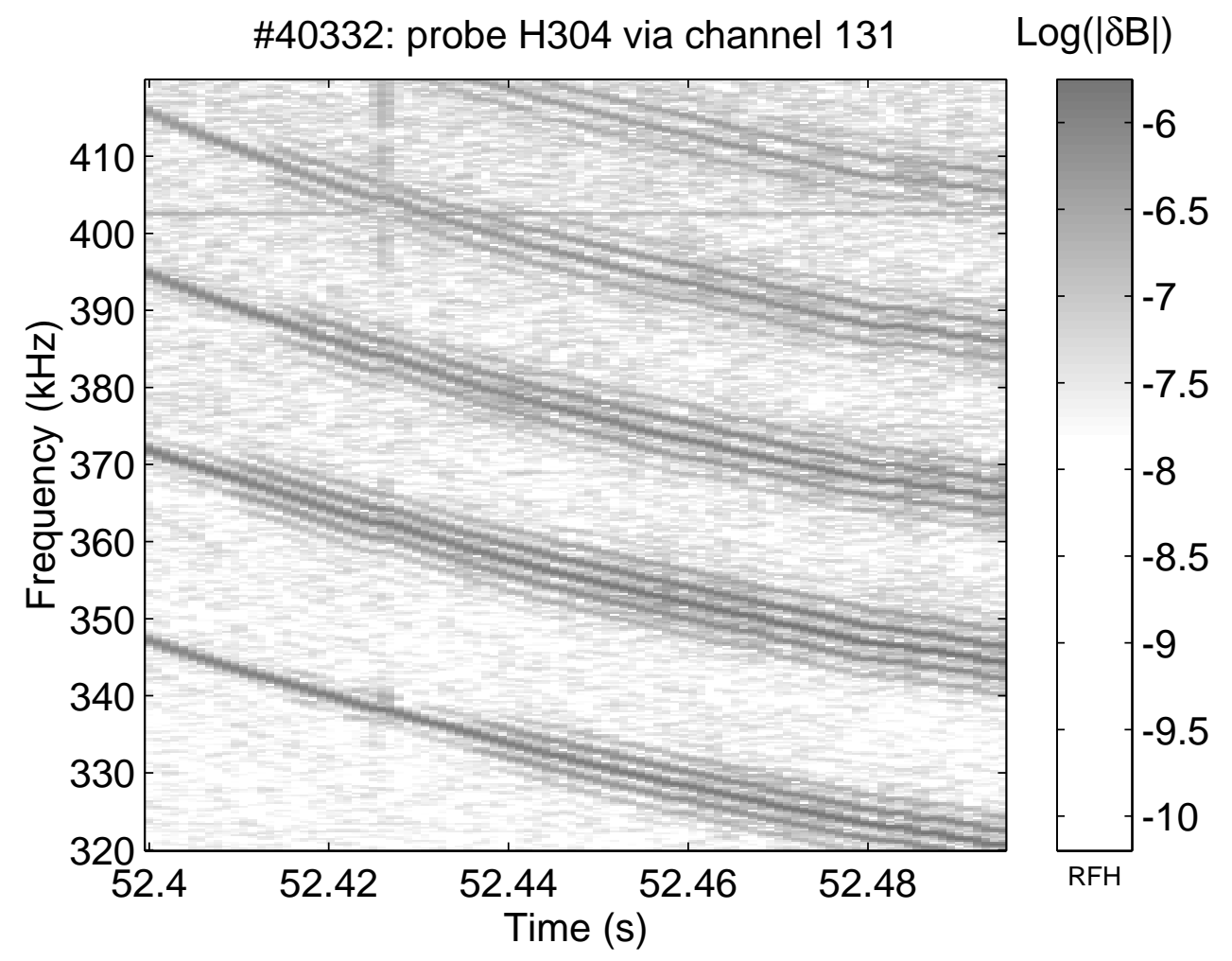

FIG. 17. Spectrogram zoom revealing magnetic fluctuation pitchfork splitting in JET Pulse 40332.

There are other alternatives to the FFT-based spectrogram. Wavelet analysis in particular would probably be very useful, but it is computationally more intensive, and therefore not as suitable as FFTs for routine processing of large amounts of data. Wavelets represent an area for future work, though, since computing capability will continue to advance whereas the amount of data needed to characterize an AE will remain constant.

Two different spectrogram routines were written in FastMag. The first provides a quick look at the data, and formats figures for rapid preparation into presentations and publications. The second one is automated for the massproduction of spectrograms for multiple probes, allowing rapid mode number analyses (see below). This latter one also includes a saturation check to be sure that the digitizers did not saturate during the relevant time window, and it can be run interactively to produce contour plots and surface plots as well as spectrograms, for detailed analyses. Both routines were written for this project by borrowing existing MATLAB library functions and then making algorithmic improvements to increase speed and reduce memory consumption.

\section{Timeslice Analyses: Mode Amplitude and Sensitivity}

In order to allow direct comparisons between the output of various probes, and to determine explicitly the amplitude of various observed signals, two different "timeslice" routines were produced, which extract the amplitude and phase vs. frequency at a single time (by slicing through the spectrogram).

The first routine looks at data for just one probe. It reports the measured fluctuations and the transfer function. The transfer function is equivalent to the fluctuation amplitude (in Tesla) corresponding to a 1-count signal. The typical digitizer noise is in fact one count, and knowing the transfer function, this routine also produces a semi-empirical noise-level estimate. The actual digitizer noise is \pm 1 count, but because the FFT averages over a large number of points (typically 2048 or 4096), the effective noise is reduced by over an order of magnitude. This technique also allows an empirical check on the calibration: during dry-run pulses random noise is piped into the digitizers and the resulting spectrum matches the empirical transfer-function-based noise-estimate curve nicely. Fig. 18 provides a timeslice from the same discharge as Fig. 17, and shows that in pitchfork splitting the side-modes are symmetrically offset in frequency from the central mode, but need not be symmetric in amplitude. 


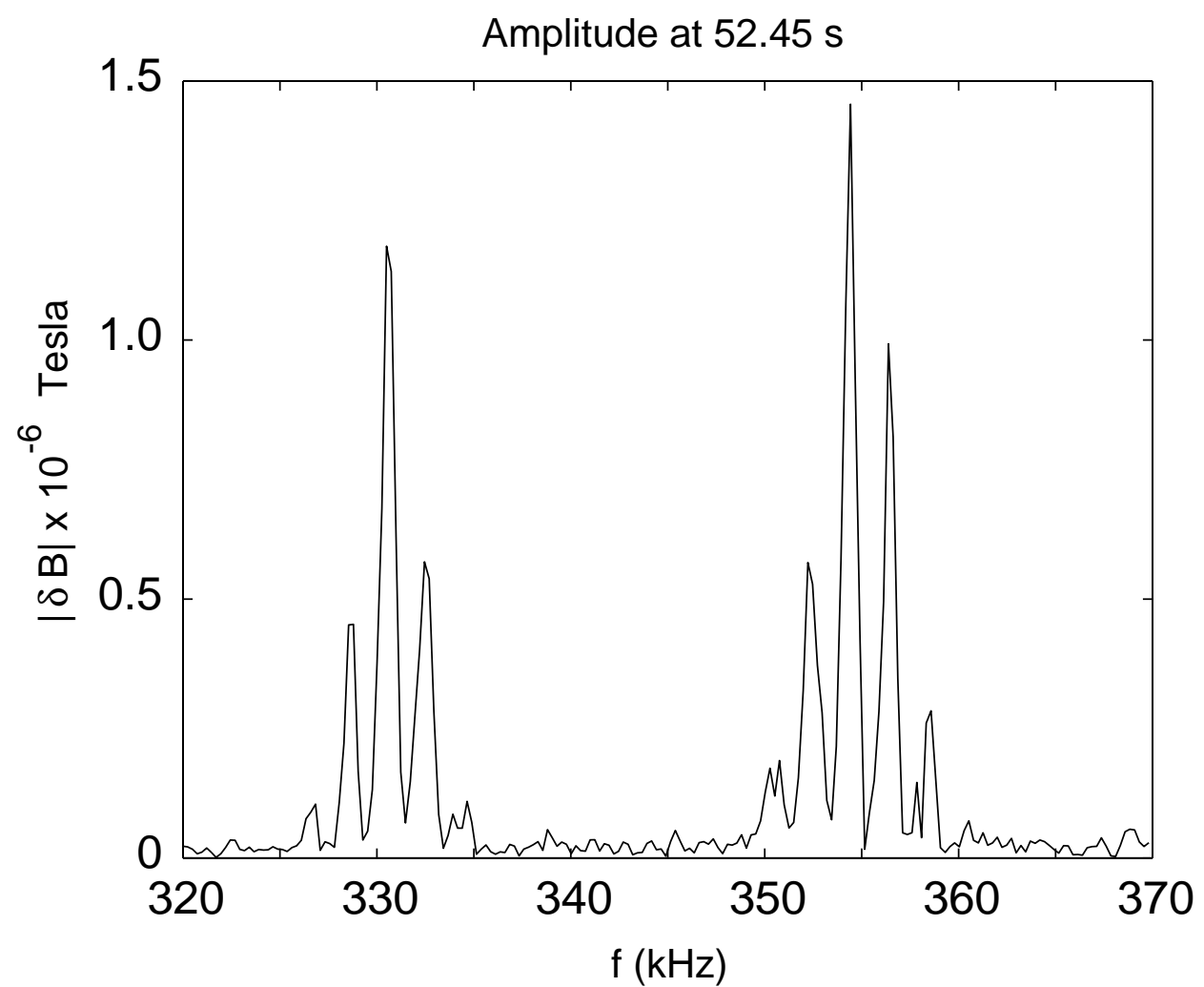

FIG. 18. Timeslice of magnetic fluctuations from $320-370 \mathrm{kHz}$ at $52.45 \mathrm{~s}$ in \#40332, analyzed with a 4096 -point (4.096 ms) FFT.

The second timeslice routine allows comparison of data from multiple probes. In addition to plotting each probe's output individually (using the first routine), it also overlays the responses of multiple probes. This allows another direct check of the amplitude-response calibration; it is found that different probes, once calibrated, produce response amplitudes that are identical to within the known calibration error. One can then go further and use this to discriminate between weak signals (appearing on all probes equally) and noise (which is generally different for each probe).

\section{Frequency Identification and Additional Heating}

There are two figures commonly desired for interpreting the KC1F spectrograms. The first of these is a frequencyidentification plot indicating the expected frequencies of the Alfvén Eigenmodes and ICRH Beatwaves. The second is a plot of the auxiliary heating power (NBI and ICRH) applied to the plasma, since these are the sources of fast ions which drive the AEs. Both types of plots require access to standard data waveforms on the IBM. A collection of MATLAB, FORTRAN and shell-script routines were developed for this project, based on existing JET libraries, to download the required types of data and rapidly and routinely produce both types of figures.

\section{Mode Number Analysis}

The most important advantage of the calibrated data over the uncalibrated data is that it allows one to compare the phases of various signals on the different probes, and thereby to extract the toroidal mode number. The basic algorithm used here is to take the phases $\alpha_{j}$ for nearby probes at toroidal angles $\phi_{j}$ and determine the mode number $n$ using the phase-slope method:

$$
n \equiv \Delta \alpha / \Delta \phi
$$


This can be done rapidly with only two probes, but phase data is often noisy (particularly for weak signals), and it is very useful to use a third probe to validate the quality of the phase slope. Rather than go to the computational expense of doing a full-blown least-squares fit on just three points of data, a simpler approach is used here. The slope is determined using the two probes with highest and lowest toroidal position. The intercept is determined by ensuring that the line passes through the "average" point (whose phase and location are the average for the dataset). The RMS error is then determined. If the error is larger than a user-controlled threshold (typically 0.2 radians) then the point is rejected as a bad fit. If the error is sufficiently low then the slope is mapped to the nearest integer, which is presumed to be the mode number.

Because the mode-number processing cannot rely on built-in MATLAB functions, it is slow compared to the spectrogram generation. To reduce the amount of number-crunching, the mode-number routine allows the user to specify a small window in time and frequency for the analysis. Then an amplitude-threshold test is applied to the data before it is analyzed. Only those data points are considered whose amplitude exceeds some fraction of the 1-count signal amplitude (typically 0.5), indicating that the signal is well above the noise (usually at about 0.1 after FFT averaging) and likely to have a meaningful phase. For points which meet the test, the phases from each of the probes are "unwrapped" (removing any jumps greater than $\pi$ by shifting points by $2 \pi$ ). Mode numbers are then produced using those points with significant amplitude and low fit errors. The mode numbers are then mapped into a color scheme and the resulting mode-number spectrogram is displayed. The error estimates for each point are also displayed. In practice one cannot always determine a mode number from a single point (due to the possible presence of noise), but in the spectrogram all the points corresponding to a given mode generally show up with nearly identical mode numbers, and one can be confident that the mode number is correct. Returning to the example of the TAEs in Figs. 16 and 17, the mode-number analysis determined that in the pitchfork-splitting phenomenon each of the "new" modes has the same toroidal mode number as the original, central mode. This led to the hypothesis that the formation of the sideband modes might be due to amplitude modulation of the TAE. This hypothesis was confirmed by bandpass-filtering the calibrated time-domain data to extract the signal from a particular mode, and then plotting the resulting time trace, as shown in Fig. 19. ${ }^{5}$

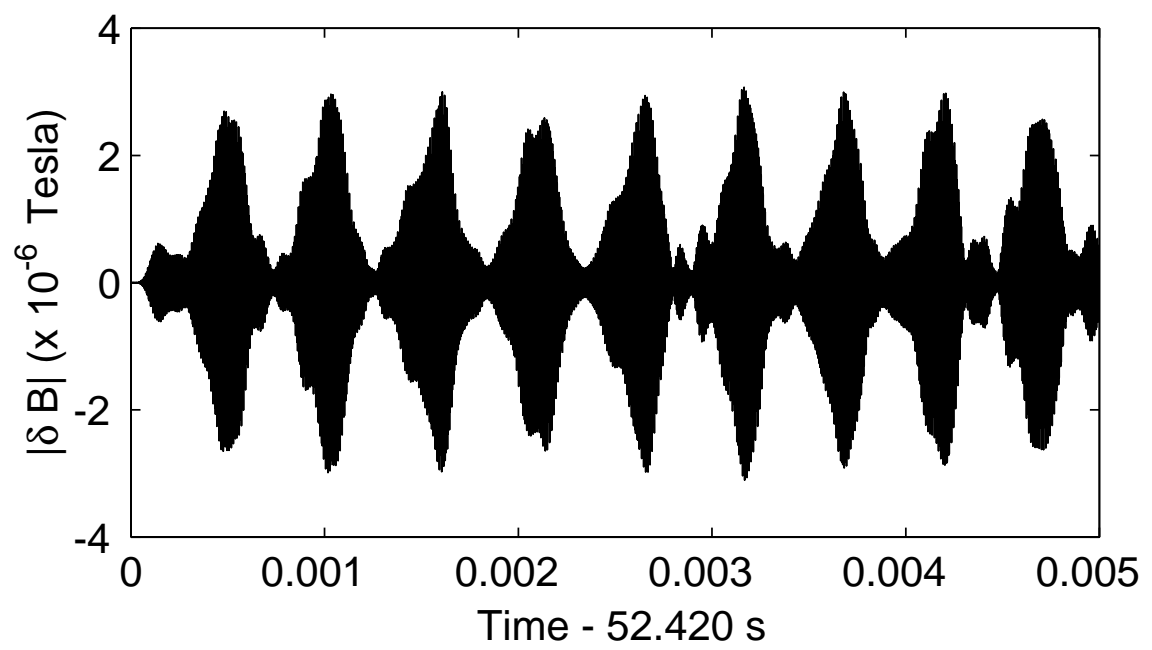

FIG. 19. Time trace of calibrated, digitally-filtered data for the $365 \mathrm{kHz} n=7$ TAE shown in Figure 17, highlighting the amplitude modulation of the signal. The filtering used a 7th-order Butterworth 355-375 kHz bandpass filter with $1 \mathrm{~dB}$ ripple and $>40 \mathrm{~dB}$ attenuation below $350 \mathrm{kHz}$ and above $380 \mathrm{kHz}$.

The range of mode numbers which can be determined is restricted by the limitation that the phase jump $\Delta \alpha$ between probes separated by a toroidal angle $\Delta \phi$ cannot be greater than $\pi$. The phase jump $\Delta \alpha$ is equivalent to $n \Delta \phi$ by equation 27 , so the limit is then $|n \Delta \phi| \leq \pi$, or

$$
|n| \leq \pi /|\Delta \phi|
$$

For the 3 probes in the JET high-resolution toroidal set (H302, H303, H304), the largest gap is 10.17 degrees (by Table I), so we can determine $-17 \leq n \leq 17$ with confidence. When higher mode numbers are needed one can use just two probes (H303 and H304), which are separated by only 5.63 degrees, and determine $-32<n<32$.

Equation 27 also allows an estimate of the error in the mode-number calculation. The toroidal positions of the coils are well known, but the phase measurements are subject to both signal noise and calibration errors. As mentioned in Section IIIE, the overall known phase error from the calibration process is roughly 1.7 degrees, with the maximum 
at certain frequencies being as large as 2.9 degrees.

For the standard 3-probe mode-number analysis the phase-slope calculation is done using probes H302 and H304, with a toroidal separation of 15.80 degrees. For strong signals, if we assume that the phase error is dominated by the known calibration errors then we can estimate the mode number error to be roughly $\Delta n \leq 5.8 / 15.80=0.37$, and the integer mode numbers that are calculated should be correct. For weaker signals, however, the phase error can increase, and if the mode number error exceeds 0.5 then the mode-number determination algorithm, which uses the nearest-integer approach, may end up off by one. These conclusions were borne out in practice, where we were able to measure mode numbers $\mathrm{n}=1,2,3,4$ of MHD activity at Doppler-shifted frequencies corresponding to $n f_{\text {rot }}$, the correct multiples of the toroidal plasma rotation frequency.

For the 2-probe high-n mode number analysis the mode number error becomes $\Delta n \leq 5.8 / 5.63=1.03$, and the mode number determination is no longer exact to better than \pm 1 or 2 . However, this method is only necessary when one needs to look at $|n|>17$, and one rarely needs an exact mode number in that case; the error amounts to only $10 \%$ or so.

\section{Automated Processing}

To further speed up the data analysis, automated batch processing routines have also been constructed, which allow the spectrogram and mode number routines to be run automatically for several probes and several pulses at once. The diagnostician can then spend his time thinking about the physics rather than repetitively giving instructions to the program.

The automated batch processing routines were subsequently used to produce an automatic overnight processing routine. This routine starts automatically at a specified time and carries out standard amplitude and mode-number analyses for all data in a given pulse range (which can include the most recent pulses). The resulting figures are written to JPEG images and HTML pages for viewing with World-Wide-Web browsers.

\section{KC1F PULSE-CHARACTERIZATION DATABASE}

In order to make systematic analyses of AE phenomena in a wide variety of JET plasma configurations, a pulsecharacterization database toolkit was developed. This section describes the database and provides an overview of the first JET campaign in which data was collected using KC1F. Although the discussion here is brief, it cannot be overstated how valuable the database was in allowing efficient analysis of the KC1F dataset. In addition to the discussion here, the technical details of the database system are provided in Appendix B of the the author's dissertation. ${ }^{5}$ Some examples of the results obtained by using this database to mine the KC1F data are included in the literature; ${ }^{1,2,5-17}$ the most detailed results to date are included only in the thesis ${ }^{5}$ but should be published shortly in the refereed literature.

Roughly 200 gigabytes of KC1F data was collected from 4280 pulses during the pre-D-T, D-T, and ITER Physics campaigns during 1997 and 1998, when JET used the Mark IIA Divertor. This span covers Pulse \#40135 of Jan. 22, 1997, to Pulse \#44414 of Feb. 2, 1998. JET performed a wide range of experiments including operation with H, D, $\mathrm{T}$ and ${ }^{3} \mathrm{He}$ gases, operation in L mode and ELM-free and ELMy H-modes, and operation with both normal profiles and Optimised Shear. ICRH heating up to $12 \mathrm{MW}$ was used, as was NBI heating up to $23 \mathrm{MW}$.

$\mathrm{KC} 1 \mathrm{~F}$ data was analyzed on a daily basis as it was acquired, and a number of interesting phenomena were revealed in that way. It was also desired to assess systematically the KC1F diagnostic observations in the various JET plasma configurations, in particular the Alfvén Eigenmode activity under various ICRH and NBI heating schemes. This top-down approach was useful partly because the bottom-up approach involving careful analysis of "interesting" discharges cannot yield definitive conclusions about the universal presence or absence of unstable modes in all pulses of a particular type.

Furthermore, to carry out studies of AEs and other high-frequency MHD phenomena, it is not enough to conquer the problem of adequate diagnostic coverage as stated in Section II B. There is also the challenge of making intelligible the hundreds of megabytes of data obtained with these diagnostics. For instance, the KC1F diagnostic acquires 64 MB of data per pulse. However, the amount of information which can be displayed at one time on a computer monitor (say 1000 by 1000 pixels) is at most 1-2 MB. Even at 300 dots per inch, which is difficult to resolve with the naked eye, a single letter-size page holds less than $8 \mathrm{MB}$ of data. Efficient analysis of a full set of high-frequency MHD data is therefore limited by the difficulty in visually analyzing all of the data, and in this time-limited (as opposed to data-limited) regime, one would like to have a means of rapidly selecting the most interesting data for a particular analysis. 
To this end, a custom database program was written with MATLAB and used as described below. In particular we sought to develop a means of extracting pulses of particular interest without excessively compromising the scope of the analysis of those pulses. This approach allows easy reinvestigation of old, partially-understood data, which often yields up new insights only after later analysis based on subsequent advances in understanding.

The database was implemented by developing a general set of Matlab database routines and then developing an automated interface to reduce the relevant JET data to elements in the new database files. The database contains 39 elements for each pulse, including data on $B_{t}$ and $I_{p} ; \mathrm{H}, \mathrm{D}$ and T concentrations; $P_{N B I}, P_{I C R H}$, and $f_{I C R H}$. Details are shown in Table II. The key element in the database is the KC1F trigger start time and corresponding acquisition window. For each pulse, all other time-dependent data are restricted to the $\mathrm{KC1F}$ acquisition time window. The data are further reduced to single points by taking time averages, maxima, or minima over the acquisition window.

Not all 4280 pulses resulted in plasmas; some are dry runs, early disruptions, soft stops, and so on. In addition, the $\mathrm{KC1F}$ system was down for a small fraction of these pulses, resulting in no acquisition. Also, on a few occasions the trigger time was set improperly and nothing of interest was collected. Despite this, the database still contains thousands of pulses with good data. A summary of the parameter ranges in the KC1F pulse database is given in Table III.

The ICRH heating is of particular interest for analying ICRH-driven AEs. The database indicates that about 1400 pulses used ICRH, with power levels $0<P_{I C R H} \leq 12 \mathrm{MW}$. Of these, about 650 have ICRH only $\left(P_{N B I}=0\right)$, roughly 160 have ICRH with weak NBI $\left(P_{N B I}<4 \mathrm{MW}\right.$, often diagnostic blips), and approximately 615 are ICRH + NBI "combined heating" pulses. Statistics on the ICRH usage vs. frequency in the period covered by the database are shown in Fig. 20.

\begin{tabular}{|c|c|c|c|c|c|}
\hline Element & Units & Type & Node1 & Node2 & Reduction \\
\hline Trigger Start & $\mathrm{s}$ & JPF & DA/C1-TC91 & TIM & direct \\
\hline Trigger End & $\mathrm{s}$ & $\mathrm{n} / \mathrm{a}$ & $\mathrm{n} / \mathrm{a}$ & $\mathrm{n} / \mathrm{a}$ & manual \\
\hline$B_{t}(0)$ & $\mathrm{T}$ & PPF & EFIT & BVAC & $\max , \min$ \\
\hline$B_{t}(0)$ (alt) & $\mathrm{T}$ & PPF & MAGN & BVAC & $\max , \min$ \\
\hline$I_{p}$ & A & JPF & DA/C1-IPLA & DAT & $\max , \min$ \\
\hline H conc. & $\mathrm{n} / \mathrm{a}$ & PPF & KS3B & HTHD & mean \\
\hline T conc. & $\mathrm{n} / \mathrm{a}$ & PPF & KS3B & TTTD & mean \\
\hline$P_{N B I}$ total & $\mathrm{W}$ & PPF & NBI & PTOT & $\max , \min$ \\
\hline$P_{N B I} 140 \mathrm{keV}$ & $\mathrm{W}$ & PPF & NBI & P140 & $\max , \min$ \\
\hline$P_{N B I} 80 \mathrm{keV}$ & $\mathrm{W}$ & PPF & NBI & P080 & $\max , \min$ \\
\hline$P_{I C R H}$ total & $\mathrm{W}$ & PPF & ICRH & PTOT & $\max , \min$ \\
\hline$P_{I C R H}$ mod. A & $\mathrm{W}$ & PPF & ICRH & PRFA & $\max$ \\
\hline$P_{I C R H} \bmod . \mathrm{B}$ & $\mathrm{W}$ & PPF & ICRH & PRFB & $\max$ \\
\hline$P_{I C R H}$ mod. $\mathrm{C}$ & $\mathrm{W}$ & PPF & ICRH & PRFC & $\max$ \\
\hline$P_{I C R H}$ mod. D & W & PPF & ICRH & PRFD & $\max$ \\
\hline$f_{I C R H} \mathrm{~A} 12$ & $\mathrm{~Hz}$ & PPF & RFA & FR12 & mean \\
\hline$f_{I C R H} \mathrm{~A} 34$ & $\mathrm{~Hz}$ & PPF & RFA & FR34 & mean \\
\hline$f_{I C R H} \mathrm{~B} 12$ & $\mathrm{~Hz}$ & PPF & RFB & FR12 & mean \\
\hline$f_{I C R H}$ B34 & $\mathrm{Hz}$ & $\mathrm{PPF}$ & RFB & FR34 & mean \\
\hline$f_{I C R H} \mathrm{C} 12$ & $\mathrm{~Hz}$ & $\mathrm{PPF}$ & $\mathrm{RFC}$ & FR12 & mean \\
\hline$f_{I C R H} \mathrm{C} 34$ & $\mathrm{~Hz}$ & PPF & RFC & FR34 & mean \\
\hline$f_{I C R H} \mathrm{D} 12$ & $\mathrm{~Hz}$ & PPF & RFD & FR12 & mean \\
\hline$f_{I C R H} \mathrm{D} 34$ & $\mathrm{~Hz}$ & PPF & RFD & FR34 & mean \\
\hline Updated & date & $\mathrm{n} / \mathrm{a}$ & $\mathrm{n} / \mathrm{a}$ & $\mathrm{n} / \mathrm{a}$ & manual \\
\hline
\end{tabular}

TABLE II. Principal parameters included in the KC1F pulse-characterization database. The ICRH data include power information for each antenna module (A,B,C,D) and frequency information for each antenna strap-pair (e.g. A12, A34). 
The database parameters can easily be used to determine a number of important pulse characteristics. Pulses with early trigger times (40-45 sec) are generally optimised shear discharges; the rest are plasmas with standard q-profiles. One can also use the RF frequency and magnetic field information to determine the ICRH resonances in the plasma, using Eq. 29.

$$
\frac{q}{m}_{r e s}=(\text { constant }) \frac{f_{I C R H}}{B_{T}(0)}
$$

This gives the ratio of charge (q) to mass $(\mathrm{m})$ for the resonant species assuming on-axis ICRH. (The constant factor is normalized to the $\mathrm{q}$ and $\mathrm{m}$ of hydrogen, so $\mathrm{H}$ has a $\mathrm{q} / \mathrm{m}$ of $1,{ }^{3} \mathrm{He}$ is $1 / 3, \mathrm{D}$ is $1 / 2$, and $\mathrm{T}$ is $1 / 3$.) One can also identify monochromatic vs. polychromatic ICRH by checking the mean frequencies of the different modules and seeing how different they are, and determine the type of NBI injection.

All of these techniques were used to characterize the AE excitation in the various classes of JET discharges during this campaign, and to identify interesting phenomena in each class. The results obtained by applying this database to the $\mathrm{KC} 1 \mathrm{~F}$ data analysis are beyond the scope of this paper, but can be found in the author's thesis ${ }^{5}$ and eventually in the literature.

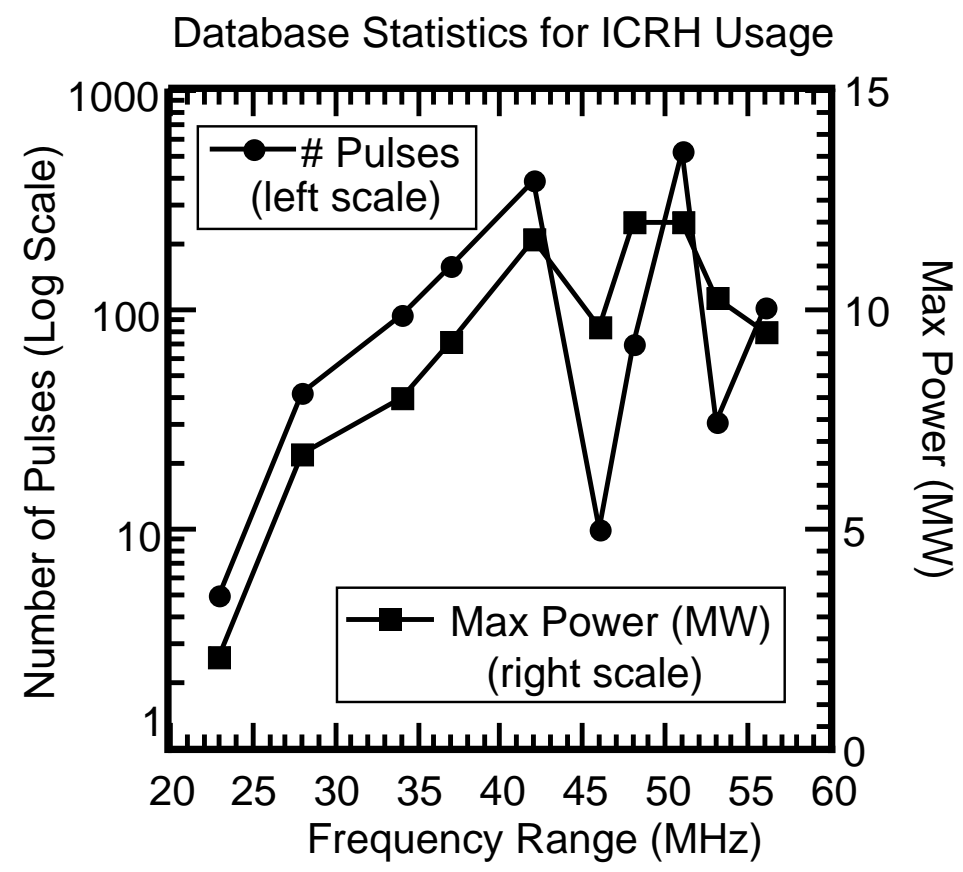

FIG. 20. ICRH Statistics for the KC1F Pulse Database. Only data during the KC1F window are used. Note: Some pulses actually had polychromatic heating (multiple frequency ranges), but the maximum power which is shown is always from monochromatic heating.

\begin{tabular}{l|l|l|l}
\hline \hline Parameter & Units & Minimum & \\
\hline Trigger Time & $\mathrm{s}$ & 40 & Maximum \\
$B_{t}(0)$ & $\mathrm{T}$ & 0.8 & 31 \\
$I_{p}$ & $\mathrm{MA}$ & 0 & 3.8 \\
$\mathrm{H}$ conc. & $\%$ & 0 & 7.8 \\
T conc. & $\%$ & 0 & $98 \%$ \\
$P_{N B I}$ total & $\mathrm{MW}$ & 0 & $95 \%$ \\
$P_{N B I} 140 \mathrm{keV}$ & $\mathrm{MW}$ & 0 & 22.3 \\
$P_{N B I} 80 \mathrm{keV}$ & $\mathrm{MW}$ & 0 & 10.9 \\
$P_{I C R H}$ total & $\mathrm{MW}$ & 0 & 12.2 \\
$f_{I C R H}$ & $\mathrm{MHz}$ & 0 & 12.0 \\
\hline \hline
\end{tabular}

TABLE III. Range of values for key parameters in the KC1F pulse-characterization database. 


\section{SUMMARY AND ACKNOWLEDGMENTS}

A new 8-channel, $4 \mathrm{~s}, 1 \mathrm{MHz}, 12$-bit data acquisition system "KC1F" has been used in conjunction with the JET fast Mirnov magnetic fluctuation pickup coils at the Joint European Torus to study Alfvén Eigenmodes and other fast magnetohydrodynamic activity. The magnetic probe coils were calibrated for the first time in the range 30-460 $\mathrm{kHz}$ using a new remote calibration technique which accounts for the presence of the first few LRC circuit resonances. A data-processing system has been developed within the MATLAB software environment to produce spectrograms of fluctuation amplitude and toroidal mode number versus frequency and time. The analysis software has been automated to allow routine overnight production of spectrogram web pages. Modes with amplitudes $\delta B / B \geq 10^{-8}$ and toroidal mode numbers $|n|<32$ can now be routinely studied. A pulse-characterization database has also been developed to select for the analysis of various useful subsets of the 4000+ JET discharges for which KC1F data is now available. Based on the work presented here and anticipated advances in data-acquisition technology, it should soon be possible to obtain complete diagnostic data on the AEs.

The authors wish to thank Prof. N. Fisch of PPPL and Drs. C. Gormezano and J. Jacquinot of JET for their support. We also thank the JET team, especially R. Felton, K. Blackler, P. Lavanchy and the Data Management Group for technical assistance, and Drs. S. E. Sharapov, D. Borba and D. Testa of JET for their contributions to operating $\mathrm{KC1F}$ and analyzing the data.

The work presented in this paper was supported in part by EURATOM and the U.S. Dept. of Energy (contract DE-AC02-76-CHO3073) through a PPPL-JET collaboration under the auspices of the IEA Three-Large-Tokamak collaboration, in part by EURATOM and the Centre des Recherches en Physique des Plasmas (CRPP) in Lausanne, Switzerland through a JET-CRPP Task Agreement, and partly by EURATOM and the U.S. Dept. of Energy (contract DE-FG02-99ER54563) through an MIT-JET collaboration. One of the authors (RFH) also acknowledges the support of the Fannie and John Hertz Foundation, and notes that the final phase of this work was performed under the auspices of the U.S. Department of Energy by University of California Lawrence Livermore National Laboratory under contract No. W-7405-Eng-48.

\section{APPENDIX A: SUPPLEMENTAL CALIBRATION INFORMATION}

This appendix contains technical reference information on the KC1F fast magnetics calibration which supplements and supports the scientific information presented in the main text.

\section{Parameter Optimization for Amplifier and Digitizer Calibration}

With regard to the amplifier-digitizer calibration (Section III B), one would like to optimize the quality of the calibration, and so it is worth considering the mathematics of the parameter choices for the frequency sweep rate and FFT.

For a signal with constant frequency, the frequency, Fourier amplitude, and phase can best be determined with a large timeslice $n_{F F T}$. This is because the frequency resolution of the FFT is the sample rate divided by the number of samples in the slice $\left(\Delta f_{F F T}=\frac{f_{s a m p l e}}{n_{F F T}}\right)$, and the error in the amplitude and phase decreases as more periods of the wave are included in the slice. However, the duration of the slice must then increase: $\Delta t_{\text {slice }}=n_{F F T} \tau_{\text {sample }}$, where $\tau_{\text {sample }}=1 / f_{\text {sample }}$. This is an illustration of the "Fourier Uncertainty Principle",

$$
\Delta f_{F F T} \Delta t_{\text {slice }}=1,
$$

which is simply a mathematical property of the discrete Fourier transform. (It is intriguing that if you multiply by Planck's constant $h$ and use $E=h f$ you can obtain a form of the Heisenberg Uncertainty Principle $\Delta E \Delta t=h$.) So if one wants to know the frequency of a signal precisely, one cannot know exactly when one is measuring it, and vice versa.

For a signal which is sweeping in frequency over time, however, there is a competing effect. The longer the duration of the slice, the more the frequency of the injected signal will change. The sweep in the frequency of the injected signal during an FFT slice, $\Delta f_{\text {sweep }}$, is given by

$$
\Delta f_{\text {sweep }}=\Delta t_{\text {slice }} \frac{f_{\text {max }}-f_{\text {min }}}{\tau_{\text {sweep }}}
$$


A useful rule of thumb is to keep $\Delta f_{\text {sweep }} \approx \Delta f_{F F T}$ (or less) so that one can be sure the frequency of the peak response determined with the FFT is in fact the same frequency that was injected (to within the FFT uncertainty). This implies that one would like

$$
\tau_{\text {sweep }} \approx\left(\Delta t_{\text {slice }}\right)^{2}\left(f_{\max }-f_{\text {min }}\right)
$$

and consequently, in order to maximize the FFT resolution with large $\Delta t_{\text {slice }}$, one would like the longest possible $\tau_{\text {sweep }}$. The first $\mathrm{KC1F}$ calibration was done when the system was only configured with $16 \mathrm{MB}$ of RAM, sufficient for only 1 second of data acquisition, so the sweep period was set to 0.5 seconds (to be sure there was a single full unbroken sweep within the 1 -second window). The corresponding optimal FFT window turns out to be $t_{\text {slice }}=1.02$ $\mathrm{ms}$, so the choice was made to use $n_{F F T}=1024$ samples for the FFT.

\section{Proof of the Theorem of Section III C 3}

This section contains a proof of the $H=\operatorname{Den}(\mathrm{Z})$ theorem of Section III C 3. Despite the simplicity of the theorem and considerable effort, the authors were unable to find a simpler proof than that presented here. To maintain clarity, the circuit model is redrawn in Fig. 21 (top) using the notation of Eq. 16. The approach is to treat $V_{\text {out }}$ and all the $X_{j}$ and $Y_{j}$ as known, and determine the inverse transfer function $1 / H=V_{i n} / V_{\text {out }}$. What we will find is that indeed the inverse transfer function is identical to the denominator $\mathrm{D}$ of the impedance.

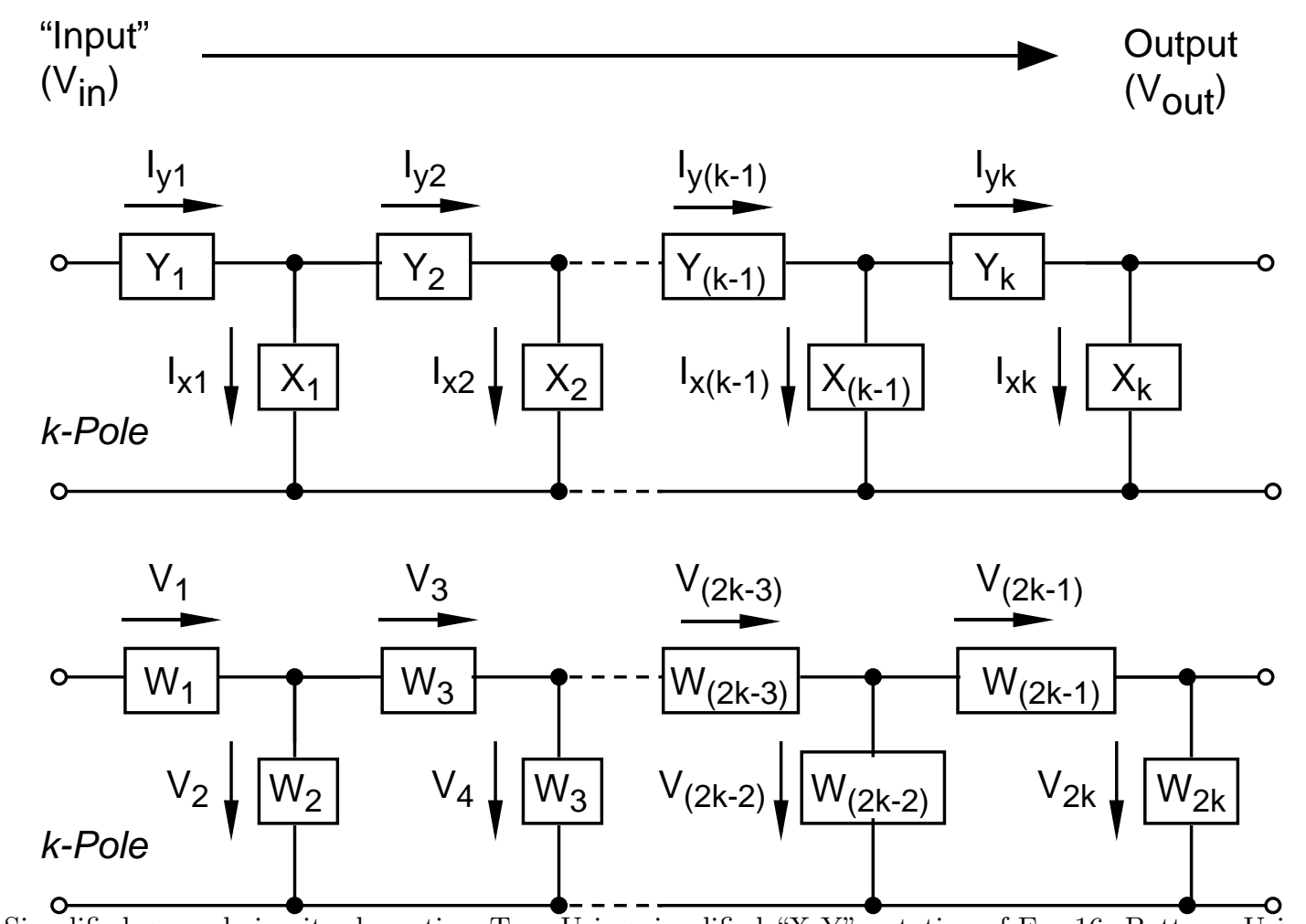

FIG. 21. Simplified general circuit schematics. Top: Using simplified "X-Y" notation of Eq. 16. Bottom: Using normalized "W" notation of Eq. A6.

Kirchhoff's equations expressing conservation of energy and charge can be used to determine the voltage and current in each segment of the circuit. The voltage-loop equations take the form $V_{j}-V_{j+1}-V_{j+2}=0$ and the current-junction equations are $I_{j}-I_{j+1}-I_{j+2}=0$. At the input end, $V_{i n}-V_{1}-V_{2}=0$, while at the output end things simplify: $V_{2 k-2}-V_{2 k-1}-V_{\text {out }}=0$ and $I_{2 k-1}-I_{2 k}=0$.

These equations lend themselves to matrix form, but first it is convenient to normalize everything to $V_{\text {out }}=I_{2 k} / X_{2 k}$ and work only with the voltages rather than the currents. Also, because the normalization depends on the order of the circuit, and we will want to compare circuits of different order later, we denote the circuit order with a prepended superscript-k. Thus: 


$$
\begin{aligned}
{ }^{k} W_{i} & = \begin{cases}Y_{\frac{i+1}{2}} X_{k}, & i=\text { odd } \\
X_{k} / X_{\frac{i}{2}}, & i=\text { even }\end{cases} \\
{ }^{k} I_{i} & = \begin{cases}I_{y\left(\frac{i+1}{2}\right)} / I_{x k}, & i=\text { odd } \\
I_{x\left(\frac{i}{2}\right)} / I_{x k}, & i=\text { even }\end{cases} \\
{ }^{k} V_{i} & ={ }^{k} I_{i}^{k} W_{i} .
\end{aligned}
$$

Note that ${ }^{k} W_{2 k}=1,{ }^{k} I_{2 k}=1$, and ${ }^{k} V_{\text {out }}=1$ in this notation. Also, normalizing $V_{i n}\left(=V_{0}\right)$ causes it to become ${ }^{k} V_{\text {in }}=V_{\text {in }} / V_{\text {out }}=1 / H_{k}$, so we just need to solve for ${ }^{k} V_{\text {in }}$. The schematic for this notation is shown in the bottom of Fig. 21.

The complete description of the $2 \mathrm{k}$ equations for the $\mathrm{k}$-th order circuit is then given by the matrix equation

$$
\left[{ }^{k} \mathbf{M}\right]\left[{ }^{k} \mathbf{V}\right]=\left[{ }^{k} \mathbf{A}\right]
$$

where ${ }^{k} \mathbf{M}$ is

$$
\left[\begin{array}{ccccccccc}
1 & -1 & -1 & 0 & 0 & \cdots & \cdots & \cdots & 0 \\
0 & W_{1}^{-1} & -W_{2}^{-1} & -W_{3}^{-1} & 0 & \cdots & \cdots & \cdots & 0 \\
0 & 0 & 1 & -1 & -1 & 0 & \cdots & \cdots & 0 \\
0 & 0 & 0 & W_{3}^{-1} & -W_{4}^{-1} & -W_{5}^{-1} & \cdots & \cdots & 0 \\
\vdots & \cdots & \cdots & \cdots & \cdots & \cdots & \cdots & \cdots & \vdots \\
0 & \cdots & \cdots & \cdots & 0 & 1 & -1 & -1 & 0 \\
0 & \cdots & \cdots & \cdots & \cdots & 0 & W_{2 k-3}^{-1} & -W_{2 k-2}^{-1} & -W_{2 k-1}^{-1} \\
0 & \cdots & \cdots & \cdots & \cdots & 0 & 0 & 1 & -1 \\
0 & \cdots & \cdots & \cdots & \cdots & 0 & 0 & 0 & W_{2 k-1}^{-1}
\end{array}\right]
$$

containing the circuit component values $W_{j}$ (presumed known), where

$$
{ }^{k} \mathbf{V}^{\mathbf{t}}=\left[{ }^{k} V_{\text {in }}{ }^{k} V_{1} \quad \cdots{ }^{k} V_{2 k-2}{ }^{k} V_{2 k-1}\right]
$$

is the (transposed) set of unknown voltages, and where

$$
{ }^{k} \mathbf{A}^{\mathbf{t}}=\left[\begin{array}{lllll}
{ }^{k} A_{1} & { }^{k} A_{1} & \cdots & { }^{k} A_{2 k-1} & { }^{k} A_{2 k}
\end{array}\right]=\left[\begin{array}{lllll}
0 & \cdots & 0 & 1 & 1
\end{array}\right]
$$

is the (transposed) vector containing the normalized constants for each of the circuit equations. (Beware that ${ }^{k} A_{2 k}$ corresponds to ${ }^{k} V_{2 k-1}$ in this notation.)

One could read off the value for ${ }^{k} V_{2 k-1}$ directly and then work up the matrix; this would yield ${ }^{k} V_{i n}$ in terms of the other ${ }^{k} V_{i}$, which in turn are functions of the ${ }^{k} W_{i}$. However, for our purposes it works better to go the other way, solving the matrix for ${ }^{k} V_{i n}$ from the top down by using Gaussian elimination to systematically cancel the non- ${ }^{k} V_{i n}$ terms in ${ }^{k} \mathbf{M}$.

Since the crux of the proof hinges on the details of the elimination process, it is necessary to detail how it goes. Starting with the second row $i=2$ of ${ }^{k} \mathbf{M}$ marching downwards $2 k-1$ times, and using the notation ${ }^{k} m_{i, j}$ for the $j^{t h}$ element of the $i^{\text {th }}$ row of ${ }^{k} \mathbf{M}$, we do these steps for each row $i$ :

Step 1 Define a rescaling factor:

$$
{ }^{k} C_{i}=-{ }^{k} m_{(i-1), i} /{ }^{k} m_{i, i}
$$

Step 2 Use ${ }^{k} C_{i}$ to rescale row i, so that the leading nonzero element is the negative of the corresponding element of row i-1. Since at most 3 elements of row i are nonzero, the effects of this step are:

$$
\begin{array}{ll}
{ }^{k} m_{i, i} & \rightarrow{ }^{k} C_{i}^{k} m_{i, i}=-{ }^{k} m_{(i-1), i} \\
{ }^{k} m_{i,(i+1)} & \rightarrow{ }^{k} C_{i}^{k} m_{i,(i+1)} \\
{ }^{k} m_{i,(i+2)} & \rightarrow{ }^{k} C_{i}^{k} m_{i,(i+2)} \\
{ }^{k} \mathbf{A}_{\mathbf{i}} & \rightarrow{ }^{k} C_{i}^{k} \mathbf{A}_{\mathbf{i}}
\end{array}
$$


Step 3 Add row i-1 to row i to cancel out that leading nonzero element and propagate the information about $V_{\text {in }}$ to the next row:

$$
\begin{aligned}
& { }^{k} m_{i, 1} \quad \rightarrow 1 \\
& { }^{k} m_{i, i} \longrightarrow 0 \\
& { }^{k} m_{i, i+1} \rightarrow{ }^{k} m_{i, i+1}+{ }^{k} m_{i-1, i+1} \\
& { }^{k} \mathbf{A}_{\mathbf{i}} \quad \rightarrow{ }^{k} \mathbf{A}_{\mathbf{i}}+{ }^{\mathbf{k}} \mathbf{A}_{\mathbf{i}-\mathbf{1}}
\end{aligned}
$$

The final result of this process will be that the bottom row of ${ }^{k} \mathbf{M}$ will consist only of a 1 in the first column, representing the equation

$$
{ }^{k} V_{\text {in }}={ }^{k} A_{2 k}^{(\text {final })} .
$$

So to determine ${ }^{k} V_{i n}$ we just need to see what the process above does to ${ }^{k} A_{2 k}$.

It should be easy to see that if ${ }^{k} \mathbf{A}$ has arbitrary elements, and we define ${ }^{k} C_{1}=1$, then the process above will rescale each ${ }^{k} A_{i}$ by the corresponding ${ }^{k} C_{i}$ and then add to it the previously-rescaled ${ }^{k} A_{i-1}$, so that finally

$$
{ }^{k} A_{2 k}^{(\text {final })}=\sum_{i=1}^{2 k}\left({ }^{k} C_{i}^{k} A_{i}^{(\text {initial })}\right) .
$$

Furthermore, the ${ }^{k} C_{i}$ can be determined recursively using the known structure of the initial ${ }^{k} \mathbf{M}$ and accounting for the effects of the algorithm above on the ${ }^{k} m_{i, i+1}$ terms. Carrying out the steps described above one can readily see that:

$$
\begin{aligned}
& { }^{k} C_{1}=1 \\
& { }^{k} C_{2}={ }^{k} W_{1} \\
& { }^{k} C_{3}={ }^{k} C_{1}+\left({ }^{k} C_{2} /{ }^{k} W_{2}\right) \\
& { }^{k} C_{4}={ }^{k} C_{2}+\left({ }^{k} C_{3}^{k} W_{3}\right) \\
& { }^{k} C_{i}=\left\{\begin{array}{ll}
{ }^{k} C_{i-2}+\left({ }^{k} C_{i-1} /{ }^{k} W_{i-1}\right) & i=\text { odd } \\
{ }^{k} C_{i-2}+\left({ }^{k} C_{i-1}^{k} W_{i-1}\right) & i=\text { even }
\end{array}\right\} \\
& { }^{k} C_{2 k-1}={ }^{k} C_{2 k-3}+\left({ }^{k} C_{2 k-2} /{ }^{k} W_{2 k-2}\right) \\
& { }^{k} C_{2 k}={ }^{k} C_{2 k-2}+\left({ }^{k} C_{2 k-1}^{k} W_{2 k-1}\right)
\end{aligned}
$$

For even $i$ the relations above imply

$$
{ }^{k} C_{i}=\sum_{j=1}^{i / 2}\left({ }^{k} C_{2 j-1}^{k} W_{2 j-1}\right)=X_{k} \sum_{j=1}^{i / 2}\left({ }^{k} C_{2 j-1} Y_{j}\right),
$$

where we have made use of the definition of $W_{i}$ and the fact that $i$ is even. We'll find later that the case $i=2 k$ is of particular interest:

$$
{ }^{k} C_{2 k}=X_{k} \sum_{j=1}^{k}\left({ }^{k} C_{2 j-1} Y_{j}\right) .
$$

Meanwhile, for $i$ odd, we can also connect back to the X-Y notation,

$$
\begin{aligned}
{ }^{k} C_{1} & =1 \\
{ }^{k} C_{3} & ={ }^{k} C_{1}+\left({ }^{k} C_{2} /{ }^{k} W_{2}\right) \\
& =1+\left({ }^{k} W_{1} /{ }^{k} W_{2}\right) \\
& =1+X_{1} Y_{1}
\end{aligned}
$$

In other words, we have seen that for any k, ${ }^{k} C_{1}=1=D_{0}$ and ${ }^{k} C_{3}=1+X_{1} Y_{1}=D_{1}$. Since this is true for $n=0$ and $n=1$, let's take the inductive leap that up to some arbitrary $\mathrm{n}^{k}{ }^{k} C_{2 n+1}=D_{n}$. Then for the case $n+1$, Eqs. A21 and A23 imply that 


$$
\begin{aligned}
{ }^{k} C_{2 n+3} & ={ }^{k} C_{2 n+1}+\left({ }^{k} C_{2 n+2} /{ }^{k} W_{2 n+2}\right) \\
& =D_{n}+\left(X_{k} \sum_{j=1}^{n+1}\left({ }^{k} C_{2 j-1} Y_{j}\right)\right) X_{n+1} / X_{k} \\
& =D_{n}+X_{n+1} \sum_{j=1}^{n+1}\left({ }^{k} D_{j-1} Y_{j}\right) \\
& =D_{n+1},
\end{aligned}
$$

where the recursion rules of Eq. 22 were used for the final step. So ${ }^{k} C_{2 n+1}=D_{n}$ is true for any n and k. Returning to the even case, Eq. A24 together with the recursion rules of Eq. 22 leads to

$$
{ }^{k} C_{2 k}=X_{k} \sum_{j=1}^{k}\left(D_{j-1} Y_{j}\right)=X_{k} N_{k} .
$$

If we now recall that all the ${ }^{k} A_{i}$ are 0 save ${ }^{k} A_{2 k-3}$ and ${ }^{k} A_{2 k-2}$, which are 1 , we can put all the pieces together and complete the proof of the Theorem:

$$
\begin{aligned}
1 / H & =V_{\text {in }} / V_{\text {out }} \\
& ={ }^{k} V_{\text {in }} \\
& ={ }^{k} A_{2 k}^{(\text {final })} \\
& =\sum_{i=1}^{2 k}\left({ }^{k} C_{i}^{k} A_{i}^{(\text {initial })}\right) \\
& ={ }^{k} C_{2 k-1}+{ }^{k} C_{2 k} \\
& =D_{k-1}+X_{k} N_{k} \\
& =D_{k}
\end{aligned}
$$

It is worth noting that for "half-order" circuits (containing $k$ parallel components $\left(X_{1}\right.$ up to $\left.X_{k}\right)$ but $k+1$ series components ( $Y_{1}$ up to $Y_{k+1}$, the addition of the extra $Y$ component alters the impedance numerator, but not the denominator. Meanwhile, the transfer function remains unchanged, which is intuitively clear because the extra series component simply adds to the very high impedance of the device measuring the voltage on the output, and little to no current flows in the final series leg of the circuit.

It would be an interesting question to see how large is the class of circuits for which the relation $H=1 / D$ holds, but this result is sufficient for our purposes.

\section{Benchmarking the Calibration Method Using a Test Circuit}

To test the calibration method and verify the codes used to implement it, a small-scale circuit board was used, with known component values and circuit layout similar to the JET probes. For several different settings of the circuit component values, the expected impedance and transfer function vs. frequency were precisely predicted using the calibration circuit model described above. Then they were measured directly and compared to the predictions. A rational function was fit to the measured impedance. The circuit parameters were extracted from the fitted impedance function and compared to the known parameters. Due to the low-impedance measurement device used for the transfer function measurement, however, it was not possible to directly compare the fitted transfer function with the measurement. Nonetheless the results provided a useful test of the calibration method and helped to validate and debug the codes used to implement it.

\section{a. Test Circuit Description and Measurement Procedure}

A suitable circuit board, the Test Matching Unit (TMU), had already been prepared by P. Lavanchy for use with the JET Saddle Coils.

Together with a series resistance $R_{t 3}$ added to the input, the test circuit could be mapped to a simplified probe circuit, as shown in Fig. 22, Fig. 23, and Table IV. 


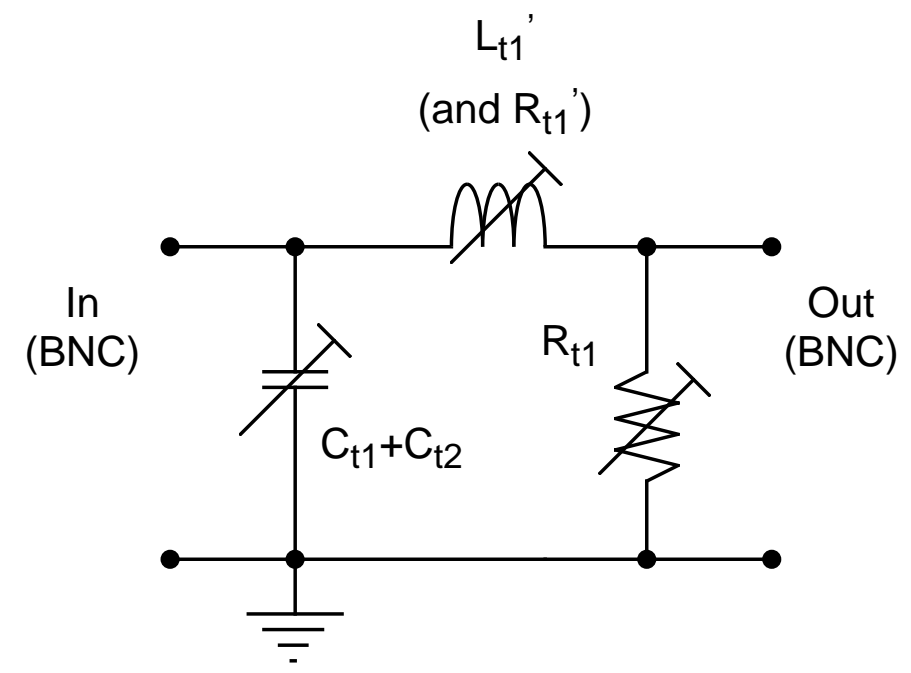

FIG. 22. Simplified schematic of Test Matching Unit emulating a probe.

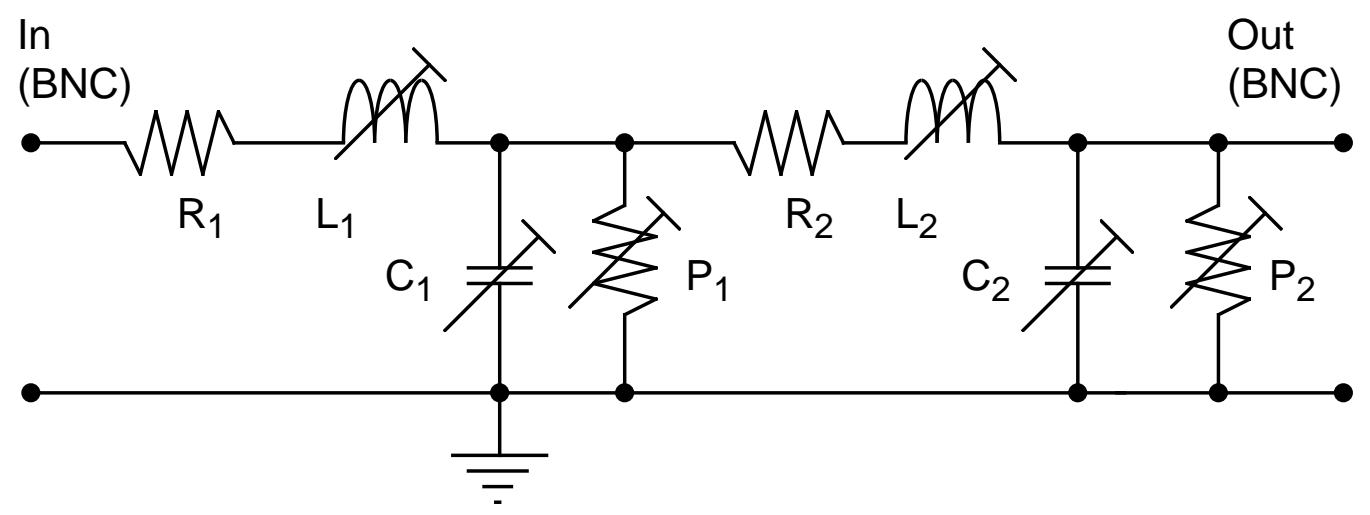

FIG. 23. Schematic of probe circuit emulated by the Test Matching Unit.

\begin{tabular}{cc}
\hline \hline TMU Notation & Probe Notation \\
\hline$R_{t 3}$ & $R_{1}$ \\
$\emptyset$ & $L_{1}$ \\
$C_{t 1}+C_{t 2}$ & $C_{1}$ \\
$\emptyset$ & $P_{1}$ \\
$R_{t 1}^{\prime}$ & $R_{2}$ \\
$L_{t 1}^{\prime}$ & $L_{2}$ \\
$\emptyset$ & $C_{2}$ \\
$1 / R_{t 1}$ & $P_{2}$ \\
\hline \hline
\end{tabular}

TABLE IV. Mapping between TMU notation and Probe Circuit Notation. 
To measure the circuit impedance vs. frequency, a Hewlett-Packard 3589A Spectrum/Network Analyzer (calibrated from $10 \mathrm{~Hz}$ to $150 \mathrm{MHz}$ ) was used with an S-parameter test set (calibrated from $100 \mathrm{kHz}$ to $150 \mathrm{MHz}$, but believed to be reliable down to $20 \mathrm{kHz}$ or so) used in Forward mode. The $50 \Omega$ source and input were used, to match the nominal impedance of the test circuit (and later the probe cables). The measurements were made using a 1:1 isolation transformer to prevent the formation of undesirable ground loops. When the real probes were measured a signal switch box was used to convert the BNC output of the Network Analyzer to the LEMO connectors used at the test point in the probe circuit. Calibration of the Network Analyzer was done using an open-circuit reflection test; this factored out the transformer and signal switch box.

For the Test Circuit measurements the frequency range tested was 20 to $1000 \mathrm{kHz}$, using a free-run trigger and 5second linear frequency sweep. The network analyzer was in the swept network mode, measuring normalized reflection coefficients to determine the impedance. The magnitude and phase of the complex $\mathrm{Z}$ were displayed on-screen, saved to floppy disks, and then transferred to a PC. Once on PC the data were converted to MATLAB format, and then analyzed using custom MATLAB routines developed for this project.

In addition to measuring the normalized impedance, the S-Parameter Test Set was also used to measure the transfer function directly, in the form $S_{21}=2 H=2\left(V_{\text {out }} / V_{\text {in }}\right)$. In this mode the input impedance of the network analyzer was only $50 \Omega$, rather than the usual high impedances of $1 \mathrm{M} \Omega$ for the network analyzer or $10 \mathrm{k} \Omega$ of the Sunnyside system. This requires making a slight modification to the theory which relates $\mathrm{Z}$ and $\mathrm{H}$, in which the high impedance of the measurement device was neglected. If we use the old notation for $N_{k}$ and $D_{k}$ (Eq. 16), and write the finite impedance of the measurement device on the output side as $Z_{\text {meas }}$, then for the Test Circuit

$$
Z_{\text {test }}=\frac{N_{\text {test }}}{D_{\text {test }}+N_{\text {test }} / Z_{\text {meas }}} .
$$

This allows an easy calculation of $\mathrm{Z}$, but one must also account for the impedance $Z_{\text {in }}$ on the input voltage (which simply adds to $\left.R_{1}\right)$. Meanwhile, from the perspective of the transfer function, $Z_{\text {meas }}$ acts as another parallel resistance on the end, changing the last admittance $\mathrm{P}$ but not affecting the fact that the transfer function $\mathrm{H}$ is just the denominator of the impedance. So now

$$
H_{\text {test }}=\frac{1}{D_{\text {test }}+N_{\text {test }} / Z_{\text {meas }}} .
$$

For the Test Circuit measurements, $R_{t 1}$ was fixed at $5.03 k \Omega$, and $C_{t 2}$ was fixed at the small value $0.5 n F$. The resistance of the inductors (denoted $R_{t 1}^{\prime}$ ) was measured for each test. The TMU settings used for the other circuit components in the four test runs are summarized in Table $\mathrm{V}$. The parameter values were chosen to be similar to the expected values of comparable parameters in the actual probes, although not all the probe parameters could be represented in the Test Circuit. For Set \#1, which had a low impedance, the network analyzer was also recalibrated using a shorted rather than open circuit, and a second impedance measurement taken for comparison.

\begin{tabular}{ccccc}
\hline \hline Set \# & $C_{t 1}(n F)$ & $L_{t 1}^{\prime}(\mu H)$ & $R_{t 1}^{\prime}(\Omega)$ & $R_{t 3}(\Omega)$ \\
\hline 1 & 10.0 & 20.1 & 1.4 & 0 \\
2 & 10.0 & 20.1 & 1.4 & 51 \\
3 & 104.5 & 20.1 & 1.4 & 51 \\
4 & 10.0 & 201 & 7.3 & 51 \\
\hline \hline
\end{tabular}

TABLE V. Parameters of the Test Circuit Measurements. 


\section{b. Predicted and Measured $Z$ and $H$}

The predicted and measured impedance, $\mathrm{Z}$, and transfer function, $\mathrm{H}$, for set \#1 were in excellent agreement with each other, except for the phase of $\mathrm{Z}$ and the magnitude of $\mathrm{Z}$ where $|Z|<0.5$. The areas of bad agreement probably arise because the open-circuit calibration (large $\mathrm{Z}$ ) breaks down where $\mathrm{Z}$ is small; the prediction and measurement for the same parameters were in much better agreement using a short-circuit (small Z) calibration, but in that case the agreement breaks down for $|Z|>0.5$, where the short-circuit calibration is no longer reasonable. For sets \#2 \#3 and \#4 the agreement between theory and measurement for these (more realistic) configurations was much better. The results for Set \#4 are shown in Figure 24.
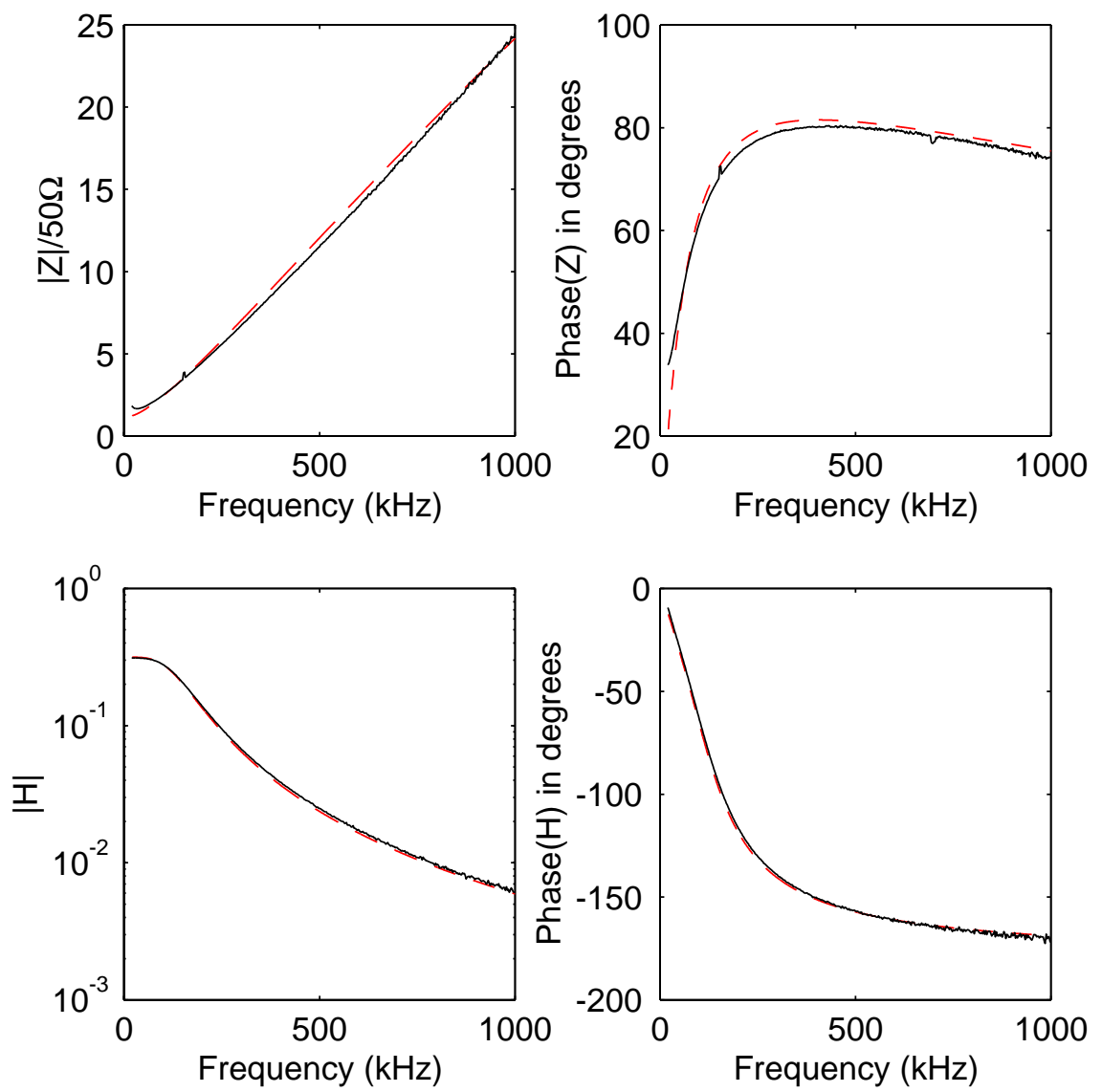

FIG. 24. Predicted and measured impedance (Z) and transfer function (H) for Test Unit, Set \#4. Both amplitude and phase are shown. An open-circuit calibration was used for the impedance measurement. The light dashed lines are the predictions, and the dark solid lines are the measurements.

On the basis of these results it is clear that the circuit model codes can accurately calculate the impedance, and that calculating the transfer function from the impedance denominator is also valid. It is worth noting that the predictions were made before the measurements were done! The main source of error so far appears to be in the impedance measurements for $f<50 \mathrm{kHz}$ (where the S-parameter Test Set calibration may be bad) and where $|Z|<0.5$ (where the open-circuit network analyzer calibration may break down).

\section{c. Rational Function Impedance Fit}

The rational impedance functions corresponding to the Test Circuit configuration have a third-order numerator (terms up to order $s^{3}$ ) and a fourth-order denominator (terms up to order $s^{4}$ ). Therefore the natural thing to do is to fit the measured impedance curves to rational functions of the same order. Because the Test Circuit is missing several components present in the equivalent Probe Circuit, however, the 3/4 fit has more free parameters than the circuit does. Furthermore, some of the impedance measurement problems described in the previous section arose at 
low frequencies, and in order to avoid these the fit was restricted to the data from $50 \mathrm{kHz}$ to $1 \mathrm{MHz}$. However, this also tends to cut out the region where the variation in $\mathrm{Z}$ is greatest, and the variation in the remaining data may not sufficiently constrain the fit. On the positive side, the fits obtained in this way match the Z data well, with RMS amplitude errors of only a few Ohms and RMS phase errors of less than 1 degree. In addition, it was found that the parameters of the dominant circuit components could in fact often be extracted from the fitted polynomials (with errors of 3-12\%), so the method does seem to work reasonably well.

In numerical tests we found for parameters of order unity that the parameter-extraction code, operating on the rational function for the impedance, extracts precisely the same circuit parameter values as those put into the impedance routine. However, the actual Test Circuits have a number of small parameters (all except for R, in fact), and numerical problems precluded the extraction of more than a few of the dominant parameters. Still, the extraction of the dominant parameters from the output end of the circuit serves as a valuable check upon the quality of the impedance fitting.

In conclusion, although the Test Circuit results did not provide a complete simulation of the calibration procedure for the actual probes, they validated key aspects of the procedure and enabled the calibration software codes to be thoroughly debugged.

${ }^{1}$ J. Jacquinot and the JET Team, Nucl. Fusion 38, 1263 (1998).

${ }^{2}$ M. Keilhacker et al., Nucl. Fusion 39, 209 (1999).

${ }^{3}$ K. L. Wong, Plasma Phys. Controlled Fusion 41, R1 (1999).

${ }^{4}$ R. F. Heeter et al., Rev. Sci. Inst. (2000), UCRL-JC-136951; Submitted.

${ }^{5}$ R. F. Heeter, Ph.D. thesis, Princeton Univ., 1999.

${ }^{6}$ A. Fasoli et al., Phys. Rev. Lett. 81, 5564 (1998).

${ }^{7}$ R. F. Heeter et al., in 5th IAEA Tech. Comm. Meeting on Alpha Particles in Fusion Research (JET Joint Undertaking, JET Joint Undertaking, Abingdon, Oxon OX14 3EA, UK, 1997), pp. 9-12.

${ }^{8}$ A. Fasoli et al., Plasma Phys. Controlled Fusion 39, B287 (1997).

${ }^{9}$ M. Keilhacker, Technical Report No. JET-P(97)55, JET Joint Undertaking, (unpublished), appeared in Proc. 17th SOFE Conference, 1997.

${ }^{10}$ P. Thomas et al., Fusion Technology 33, 407 (1998).

${ }^{11}$ W. Kerner et al., Nucl. Fusion 38, 1315 (1998).

12 D. F. H. Start et al., Plasma Phys. Controlled Fusion 40, A87 (1998).

${ }^{13}$ S. E. Sharapov et al., Technical Report No. JET-P(98)29, JET Joint Undertaking, (unpublished), Proc. Varenna Workshop on Theory of Fusion Plasmas, 1998.

${ }^{14}$ S. E. Sharapov et al., Nucl. Fusion 39, 373 (1999), also published as JET-P(98)48.

${ }^{15}$ D. F. H. Start et al., Nucl. Fusion 39, 321 (1999), also published as JET-P(98)51.

${ }^{16}$ M. Keilhacker, Philos. Trans Royal Soc. London Series A 357, 415 (1999), also published as JET report JET-P(98)70.

17 J. Jacquinot, Plasma Phys. Controlled Fusion 3A, A13 (1999).

${ }^{18}$ H. Alfvén, Arkiv. Mat. Astron. Fysik 29B, (1942).

19 J. P. Freidberg, Ideal Magnetohydrodynamics (Plenum Press, New York, 1987).

${ }^{20}$ T. H. Stix, Waves in Plasmas, 2nd ed. (American Institute of Physics, New York, 1992).

${ }^{21}$ K. Appert et al., J. Plasma Phys. 24, (1982).

${ }^{22}$ D. W. Ross et al., Phys. Fluids 25, (1982).

${ }^{23}$ C. Z. Cheng, L. Chen, and M. S. Chance, Ann. Physics 161, 21 (1985).

${ }^{24}$ C. Z. Cheng and M. S. Chance, Phys. Fluids 29, 3695 (1986).

${ }^{25}$ D. Borba et al., in Proc. 5th IAEA Technical Committee Meeting on Alpha Particles in Fusion Research (JET Joint Undertaking, JET, Abingdon, UK, 1997).

${ }^{26}$ A. Jaun et al., Plasma Phys. Controlled Fusion 39, 549 (1997).

27 S. D. Pinches, Ph.D. thesis, University of Nottingham, UK, 1996.

${ }^{28}$ M. N. Rosenbluth and P. H. Rutherford, Phys. Rev. Lett. 34, 1428 (1975).

${ }^{29}$ A. B. Mikhailovskii, Sov. Phys. JETP 41, 890 (1975).

${ }^{30}$ G. Y. Fu and J. W. V. Dam, Phys. Fluids B 1, 1949 (1989).

31 D. J. Sigmar et al., Phys. Fluids B 4, 1506 (1992).

${ }^{32}$ R. B. White et al., Phys. Plasmas 2, 2871 (1995).

${ }^{33}$ S. J. Zweben, Nucl. Fusion 38, 1345 (1998).

${ }^{34}$ M. C. Herrmann, Ph.D. thesis, Princeton Univ., 1998. 
${ }^{35}$ M. C. Herrmann and N. J. Fisch, Phys. Rev. Lett. 79, 1495 (1997).

${ }^{36}$ I. H. Hutchinson, Principles of Plasma Diagnostics (Cambridge University Press, Cambridge, UK, 1987).

${ }^{37}$ K. W. Gentle, Rev. Mod. Phys. 67, 809 (1995).

38 JET Joint Undertaking Progress Report 1997, JET Joint Undertaking, Abingdon, UK, 1998, eUR-JET-PR15.

${ }^{39}$ A. Fasoli et al., Phys. Rev. Lett. 75, 645 (1995).

${ }^{40}$ A. Fasoli et al., Phys. Rev. Lett. 76, 1067 (1996).

${ }^{41}$ A. Fasoli et al., Nucl. Fusion 36, 258 (1996).

${ }^{42}$ K. Blackler and A. W. Edwards, IEEE Trans. on Nuclear Science 41, 111 (1994).

${ }^{43}$ A. Fasoli and S. Ali-Arshad, Private Communication, 1997.

${ }^{44}$ R. Nazikian et al., Phys. Rev. Lett. 78, 2976 (1997).

${ }^{45}$ J. M. Moret et al., Rev. Sci. Inst. 69, 2333 (1998), also see ref. 3 cited herein.

${ }^{46}$ MATLAB, The Language of Technical Computing: Using MATLAB, 5th ed., The MathWorks, Inc., Natick, MA, 1997.

${ }^{47}$ MATLAB Signal Processing Toolbox User's Guide, 5th ed., The MathWorks, Inc., Natick, MA, 1997.

${ }^{48}$ R. Felton, Private Communication, 1997. 